ISSN 1855-3966 (printed edn.), ISSN 1855-3974 (electronic edn.)

\author{
ARS MATHEMATICA CONTEMPORANEA 17 (2019) 319-347 \\ https://doi.org/10.26493/1855-3974.1516.58f \\ (Also available at http://amc-journal.eu)
}

\title{
On graphs with exactly two positive eigenvalues*
}

\author{
Fang Duan ${ }^{\dagger}$ \\ College of Mathematics and Systems Science, Xinjiang University, Urumqi, P. R. China \\ School of Mathematics Science, Xinjiang Normal University, Urumqi, P. R. China \\ Qiongxiang Huang $\ddagger$ \\ College of Mathematics and Systems Science, Xinjiang University, Urumqi, P. R. China \\ Xueyi Huang $\S$ \\ School of Mathematics and Statistics, Zhengzhou University, Zhengzhou, P. R. China
}

Received 27 October 2017, accepted 28 May 2019, published online 30 October 2019

\begin{abstract}
The inertia of a graph $G$ is defined to be the triplet $\operatorname{In}(G)=(p(G), n(G), \eta(G))$, where $p(G), n(G)$ and $\eta(G)$ are the numbers of positive, negative and zero eigenvalues (including multiplicities) of the adjacency matrix $A(G)$, respectively. Traditionally $p(G)$ (resp. $n(G)$ ) is called the positive (resp. negative) inertia index of $G$. In this paper, we introduce three types of congruent transformations for graphs that keep the positive inertia index and negative inertia index. By using these congruent transformations, we determine all graphs with exactly two positive eigenvalues and one zero eigenvalue.
\end{abstract}

Keywords: Congruent transformation, positive (negative) inertia index, nullity.

Math. Subj. Class.: 05C50

* The authors are grateful to the anonymous referees for their useful and constructive comments, which have considerably improved the presentation of this paper.

†Supported by the Scientific Research Projects of Universities in Xinjiang Province (No. XJEDU2019Y030).

$\ddagger$ Corresponding author. Supported by the National Natural Science Foundation of China (Nos. 11671344, 11531011).

${ }^{\S}$ Supported by the China Postdoctoral Science Foundation (No. 2019M652556), and the Postdoctoral Research Sponsorship in Henan Province (No. 1902011).

E-mail addresses: fangbing327@126.com (Fang Duan), huangqx@xju.edu.cn (Qiongxiang Huang), huangxy@zzu.edu.cn (Xueyi Huang)

()( This work is licensed under https://creativecommons.org/licenses/by/4.0/ 


\section{Introduction}

All graphs considered here are undirected and simple. For a graph $G$, let $V(G)$ and $E(G)$ denote the vertex set and edge set of $G$, respectively. The order of $G$ is the number of vertices of $G$, denoted by $|G|$. For $v \in V(G)$, we denote by $N_{G}(v)=\{u \in V(G) \mid u v \in$ $E(G)\}$ the neighborhood of $v, N_{G}[v]=N_{G}(v) \cup\{v\}$ the closed neighborhood of $v$ and $d(v)=\left|N_{G}(v)\right|$ the degree of $v$. A vertex of $G$ is said to be pendant if it has degree 1 . By $\delta(G)$ we mean the minimum degree of vertices of $G$. As usual, we denote by $G+H$ the disjoint union of two graphs $G$ and $H, K_{n_{1}, \ldots, n_{l}}$ the complete multipartite graph with $l$ parts of sizes $n_{1}, \ldots, n_{l}$, and $K_{n}, C_{n}, P_{n}$ the complete graph, cycle, path on $n$ vertices, respectively.

The adjacency matrix of $G$, denoted by $A(G)=\left(a_{i j}\right)$, is the square matrix with $a_{i j}=1$ if $v_{i}$ and $v_{j}$ are adjacent, and $a_{i j}=0$ otherwise. Clearly, $A(G)$ is a symmetric matrix with zeros on the diagonal, and thus all the eigenvalues of $A(G)$ are real, which are defined to be the eigenvalues of $G$. The multiset consisting of eigenvalues along with their multiplicities is called the spectrum of $G$ denoted by $\operatorname{Spec}(G)$. To characterize graphs in terms of their eigenvalues has always been of the great interests for researchers, for instance to see [2, 4, $5,8,9]$ and references therein.

The inertia of a graph $G$ is defined as the triplet $\operatorname{In}(G)=(p(G), n(G), \eta(G))$, where $p(G), n(G)$ and $\eta(G)$ are the numbers of positive, negative and zero eigenvalues (including multiplicities) of $G$, respectively. Traditionally $p(G)$ (resp. $n(G)$ ) is called the positive (resp. negative) inertia index of $G$ and $\eta(G)$ is called the nullity of $G$. Obviously, $p(G)+$ $n(G)=r(G)=n-\eta(G)$ if $G$ has $n$ vertices, where $r(G)$ is the rank of $A(G)$. Let $B$ and $D$ be two real symmetric matrices of order $n$. Then $D$ is called congruent to $B$ if there is an real invertible matrix $C$ such that $D=C^{T} B C$. Traditionally we say that $D$ is obtained from $B$ by congruent transformation. The famous Sylvester's law of inertia states that the inertia of two matrices is unchanged by congruent transformation.

Since the adjacency matrix $A(G)$ of $G$ has zero diagonal, we have $p(G) \geq 1$ if $G$ has at least one edge. One of the attractive problems is to characterize those graphs with a few positive eigenvalues. In [9] Smith characterized all graphs with exactly one positive eigenvalue. Recently, Oboudi [6] completely determined the graphs with exactly two nonnegative eigenvalues, i.e., those graphs satisfying $p(G)=1$ and $\eta(G)=1$ or $p(G)=2$ and $\eta(G)=0$.

In this paper, we introduce three types congruent transformations for graphs. By using these congruent transformations and Oboudi's results in [6], we completely characterize the graphs satisfying $p(G)=2$ and $\eta(G)=1$.

\section{Preliminaries}

In this section, we will introduce some notions and lemmas for the latter use.

Theorem 2.1 (Interlacing theorem [1]). Let $G$ be a graph of order $n$ and $H$ be an induced subgraph of $G$ with order $m$. Suppose that $\lambda_{1}(G) \geq \cdots \geq \lambda_{n}(G)$ and $\lambda_{1}(H) \geq \cdots \geq$ $\lambda_{m}(H)$ are the eigenvalues of $G$ and $H$, respectively. Then for every $1 \leq i \leq m, \lambda_{i}(G) \geq$ $\lambda_{i}(H) \geq \lambda_{n-m+i}(G)$.

Lemma 2.2 ([1]). Let $H$ be an induced subgraph of graph $G$. Then $p(H) \leq p(G)$. 
Lemma 2.3 ([3]). Let $G$ be a graph containing a pendant vertex, and let $H$ be the induced subgraph of $G$ obtained by deleting the pendant vertex together with the vertex adjacent to it. Then $p(G)=p(H)+1, n(G)=n(H)+1$ and $\eta(G)=\eta(H)$.

Lemma 2.4 (Sylvester's law of inertia). If two real symmetric matrices $A$ and $B$ are congruent, then they have the same positive (resp., negative) inertia index, the same nullity.

Theorem 2.5 ([9]). A graph has exactly one positive eigenvalue if and only if its nonisolated vertices form a complete multipartite graph.

Let $G_{1}$ be a graph containing a vertex $u$ and $G_{2}$ be a graph of order $n$ that is disjoint from $G_{1}$. For $1 \leq k \leq n$, the $k$-joining graph of $G_{1}$ and $G_{2}$ with respect to $u$, denoted by $G_{1}(u) \odot{ }^{k} G_{2}$, is a graph obtained from $G_{1} \cup G_{2}$ by joining $u$ to arbitrary $k$ vertices of $G_{2}$. By using the notion of $k$-joining graph, Yu et al. [11] completely determined the connected graphs with at least one pendant vertex that have positive inertia index 2 .

Theorem 2.6 ([11]). Let $G$ be a connected graph with pendant vertices. Then $p(G)=2$ if and only if $G \cong K_{1, r}(u) \odot^{k} K_{n_{1}, \ldots, n_{l}}$, where $u$ is the center of $K_{1, r}$ and $1 \leq k \leq$ $n_{1}+\cdots+n_{l}$.

Theorem 2.7 ([6]). Let $G$ be a graph of order $n \geq 2$ with eigenvalues $\lambda_{1}(G) \geq \cdots \geq$ $\lambda_{n}(G)$. Assume that $\lambda_{3}(G)<0$, then the following hold:

(1) If $\lambda_{1}(G)>0$ and $\lambda_{2}(G)=0$, then $G \cong K_{1}+K_{n-1}$ or $G \cong K_{n} \backslash$ efor $e \in E\left(K_{n}\right)$;

(2) If $\lambda_{1}(G)>0$ and $\lambda_{2}(G)<0$, then $G \cong K_{n}$.

Let $\mathcal{H}$ be set of all graphs satisfying $\lambda_{2}(G)>0$ and $\lambda_{3}(G)<0$ (in other words, $p(G)=2$ and $\eta(G)=0$ ). Oboudi [6] determined all the graphs of $\mathcal{H}$. To give a clear description of this characterization, we introduce the class of graphs $G_{n}$ defined in [6].

For every integer $n \geq 2$, let $K_{\left\lceil\frac{n}{2}\right\rceil}$ and $K_{\left\lfloor\frac{n}{2}\right\rfloor}$ be two disjoint complete graphs with vertex set $V=\left\{v_{1}, \ldots, v_{\left\lceil\frac{n}{2}\right\rceil}\right\}$ and $W=\left\{w_{1}, \ldots, w_{\left\lfloor\frac{n}{2}\right\rfloor}\right\} . G_{n}$ is defined to be the graph obtained from $K_{\left\lceil\frac{n}{2}\right\rceil}$ and $K_{\left\lfloor\frac{n}{2}\right\rfloor}$ by adding some edges distinguishing whether $n$ is even or not below:

(1) If $n$ is even, then add some new edges to $K_{\frac{n}{2}}+K_{\frac{n}{2}}$ satisfying

$$
\begin{aligned}
\emptyset=N_{W}\left(v_{1}\right) \subset N_{W}\left(v_{2}\right)=\left\{w_{\frac{n}{2}}\right\} \subset N_{W}\left(v_{3}\right)=\left\{w_{\frac{n}{2}}, w_{\frac{n}{2}-1}\right\} \subset \cdots \subset \\
N_{W}\left(v_{\frac{n}{2}-1}\right)=\left\{w_{\frac{n}{2}}, \ldots, w_{3}\right\} \subset N_{W}\left(v_{\frac{n}{2}}\right)=\left\{w_{\frac{n}{2}}, \ldots, w_{2}\right\} .
\end{aligned}
$$

(2) If $n$ is odd, then add some new edges to $K_{\frac{n+1}{2}}+K_{\frac{n-1}{2}}$ satisfying

$$
\begin{array}{r}
\emptyset=N_{W}\left(v_{1}\right) \subset N_{W}\left(v_{2}\right)=\left\{w_{\frac{n-1}{2}}\right\} \subset N_{W}\left(v_{3}\right)=\left\{w_{\frac{n-1}{2}}, w_{\frac{n-1}{2}-1}\right\} \subset \cdots \subset \\
N_{W}\left(v_{\frac{n+1}{2}-1}\right)=\left\{w_{\frac{n-1}{2}}, \ldots, w_{2}\right\} \subset N_{W}\left(v_{\frac{n+1}{2}}\right)=\left\{w_{\frac{n-1}{2}}, \ldots, w_{1}\right\}
\end{array}
$$

By deleting the maximum (resp. minimum) degree vertex from $G_{n+1}$ if $n$ is an even (resp. odd), we obtain $G_{n}$. It follows the result below.

Remark 2.8 (See [6]). $G_{n}$ is an induced subgraph of $G_{n+1}$ for every $n \geq 2$. 


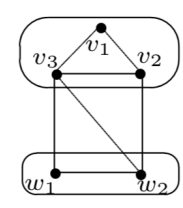

$G_{5}$

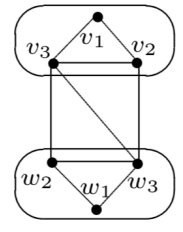

$G_{6}$

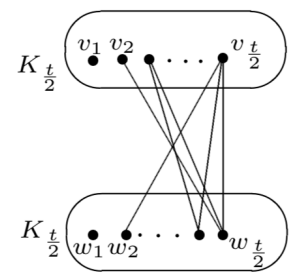

$G_{t}$

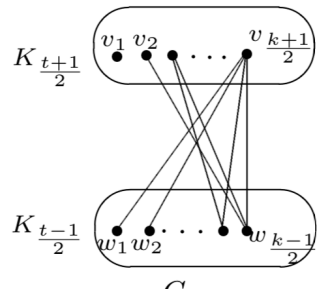

$G_{t+1}$

Figure 1: $G_{5}, G_{6}, G_{t}$ and $G_{t+1}$.

For example, $G_{2} \cong 2 K_{1}, G_{3} \cong P_{3}$ and $G_{4} \cong P_{4}$. The graphs $G_{5}$ and $G_{6}$ are shown in Figure 1. In general, $G_{t}$ and $G_{t+1}$ are also shown in Figure 1 for an even number $t$.

Let $G$ be a graph with vertex set $\left\{v_{1}, \ldots, v_{n}\right\}$. By $G\left[K_{t_{1}}, \ldots, K_{t_{n}}\right]$ we mean the generalized lexicographic product of $G$ (by $K_{t_{1}}, K_{t_{2}}, \ldots, K_{t_{n}}$ ), which is the graph obtained from $G$ by replacing the vertex $v_{j}$ with $K_{t_{j}}$ and connecting each vertex of $K_{t_{i}}$ to each vertex of $K_{t_{j}}$ if $v_{i}$ is adjacent to $v_{j}$ in $G$.

Theorem 2.9 ([6]). Let $G \in \mathcal{H}$ of order $n \geq 4$ with eigenvalues $\lambda_{1}(G) \geq \cdots \geq \lambda_{n}(G)$.

(1) If $G$ is disconnected, then $G \cong K_{p}+K_{q}$ for some integers $p, q \geq 2$;

(2) If $G$ is connected, there exist some positive integers $s$ and $t_{1}, \ldots, t_{s}$ such that $G \cong$ $G_{s}\left[K_{t_{1}}, \ldots, K_{t_{s}}\right]$ where $3 \leq s \leq 12$ and $t_{1}+\cdots+t_{s}=n$.

Furthermore, Oboudi gave all the positive integers $t_{1}, \ldots, t_{s}$ such that $G_{s}\left[K_{t_{1}}, \ldots\right.$, $\left.K_{t_{s}}\right] \in \mathcal{H}$ in Theorems $3.4-3.14$ of [6].

Let $\mathcal{G}$ be the set of all graphs with positive inertia index $p(G)=2$ and nullity $\eta(G)=1$. In next section, we introduce some new congruent transformations for graph that keep to the positive inertia index. By using such congruent transformations we characterize those graphs in $\mathcal{G}$ based on $\mathcal{H}$.

\section{Three congruent transformations of graphs}

In this section, we introduce three types of congruent transformations for graphs.

Lemma 3.1 ([10]). Let $u, v$ be two non-adjacent vertices of a graph $G$. If $u$ and $v$ have the same neighborhood, then $p(G)=p(G-u), n(G)=n(G-u)$ and $\eta(G)=\eta(G-u)+1$.

Remark 3.2. Two non-adjacent vertices $u$ and $v$ are said to be congruent vertices of I-type if they have the same neighbors. Lemma 3.1 implies that if one of congruent vertices of I-type is deleted from a graph then the positive and negative inertia indices left unchanged, but the nullity reduces just one. Conversely, if we add a new vertex that joins all the neighbors of some vertex in a graph (briefly we refer to add a vertex of I-type in what follows) then the positive and negative inertia indices left unchanged, but the nullity adds just one. The graph transformation of deleting or adding vertices of I-type is called the (graph) transformation of I-type.

Since $\operatorname{Spec}\left(K_{s}\right)=\left[(s-1)^{1},(-1)^{s-1}\right]$. By applying the transformation of I-type, we can simply find the inertia of $K_{n_{1}, n_{2}, \ldots, n_{s}}$. 
Corollary 3.3. Let $G=K_{n_{1}, n_{2}, \ldots, n_{s}}$ be a multi-complete graph where $n_{1} \geq n_{2} \geq \cdots \geq$ $n_{s}$ and $i_{0}=\min \left\{1 \leq i \leq s \mid n_{i} \geq 2\right\}$. Then $G$ has the inertia index: $\operatorname{In}(G)=$ $(p(G), \eta(G), n(G))=\left(1, n_{i_{0}}+n_{i_{0}+1}+\cdots+n_{s}-s+i_{0}-1, s-1\right)$.

The following transformation was mentioned in [4], but the author didn't prove the result. For the completeness we give a proof below.

Lemma 3.4. Let $\{u, v, w\}$ be an independent set of a graph $G$. If $N(u)$ is a disjoint union of $N(v)$ and $N(w)$, then $p(G)=p(G-u), n(G)=n(G-u)$ and $\eta(G)=\eta(G-u)+1$.

Proof. Since $u, v, w$ are not adjacent to each other, we may assume that $\left(0,0,0, \alpha^{T}\right)$, $\left(0,0,0, \beta^{T}\right)$ and $\left(0,0,0, \gamma^{T}\right)$ are the row vectors of $A(G)$ corresponding to the vertices $u, v, w$, respectively. Thus $A(G)$ can be written as

$$
A(G)=\left(\begin{array}{cccc}
0 & 0 & 0 & \alpha^{T} \\
0 & 0 & 0 & \beta^{T} \\
0 & 0 & 0 & \gamma^{T} \\
\alpha & \beta & \gamma & A(G-u-v-w)
\end{array}\right)
$$

Since $N(u)=N(v) \cup N(w)$ and $N(v) \cap N(w)=\emptyset$, we have $\alpha=\beta+\gamma$. By letting the $u$-th row (resp. $u$-th column) minus the sum of the $v$-th and $w$-th rows (resp. the sum of the $v$-th and $w$-th columns) of $A(G)$, we get that $A(G)$ is congruent to

$$
\left(\begin{array}{cccc}
0 & 0 & 0 & \mathbf{0}^{T} \\
0 & 0 & 0 & \beta^{T} \\
0 & 0 & 0 & \gamma^{T} \\
\mathbf{0} & \beta & \gamma & A(G-u-v-w)
\end{array}\right)=\left(\begin{array}{cc}
0 & \mathbf{0}^{T} \\
\mathbf{0} & A(G-u)
\end{array}\right)
$$

Thus $p(G)=p(G-u), n(G)=n(G-u)$ and $\eta(G)=\eta(G-u)+1$ by Lemma 2.4.

Remark 3.5. The vertex $u$ is said to be a congruent vertex of II-type if there exist two nonadjacent vertices $v$ and $w$ such that $N(u)$ is a disjoint union of $N(v)$ and $N(w)$. Lemma 3.4 implies that if one congruent vertex of II-type is deleted from a graph then the positive and negative indices left unchanged, but the nullity reduces just one. Conversely, if there exist two non-adjacent vertices $v$ and $w$ such that $N(v)$ and $N(w)$ are disjoint, we can add a new vertex $u$ that joins all the vertices in $N(v) \cup N(w)$ (briefly we refer to add a vertex of II-type in what follows), then the positive and negative inertia indices left unchanged, but the nullity adds just one. The graph transformation of deleting or adding vertices of II-type is called the (graph) transformation of II-type.

An induced quadrangle $C_{4}=u v x y$ of $G$ is called congruent if there exists a pair of independent edges, say $u v$ and $x y$ in $C_{4}$, such that $N(u) \backslash\{v, y\}=N(v) \backslash\{u, x\}$ and $N(x) \backslash\{y, v\}=N(y) \backslash\{x, u\}$, where $u v$ and $x y$ are called a pair of congruent edges of $C_{4}$. We call the vertices in a congruent quadrangle the congruent vertices of III-type.

Lemma 3.6. Let $u$ be a congruent vertex of III-type in a graph $G$. Then $p(G)=p(G-u)$, $n(G)=n(G-u)$ and $\eta(G)=\eta(G-u)+1$.

Proof. Let $C_{4}=u v x y$ be the congruent quadrangle of $G$ containing the congruent vertex $u$. Then $\left(0,1,0,1, \alpha^{T}\right),\left(1,0,1,0, \alpha^{T}\right),\left(0,1,0,1, \beta^{T}\right),\left(1,0,1,0, \beta^{T}\right)$ are the row vectors 
of $A(G)$ corresponding to the vertices $u, v, x$ and $y$, respectively. Thus $A(G)$ can be presented by

$$
A(G)=\left(\begin{array}{ccccc}
0 & 1 & 0 & 1 & \alpha^{T} \\
1 & 0 & 1 & 0 & \alpha^{T} \\
0 & 1 & 0 & 1 & \beta^{T} \\
1 & 0 & 1 & 0 & \beta^{T} \\
\alpha & \alpha & \beta & \beta & A(G-u-v-x-y)
\end{array}\right) .
$$

By letting the $u$-th row (resp. $u$-th column) minus the $x$-th row (resp. $x$-th column) of $A(G)$, and letting the $v$-th row (resp. $v$-th column) minus the $y$-th row (resp. $y$-th column) of $A(G)$, we obtain that $A(G)$ is congruent to

$$
B=\left(\begin{array}{ccccc}
0 & 0 & 0 & 0 & \alpha^{T}-\beta^{T} \\
0 & 0 & 0 & 0 & \alpha^{T}-\beta^{T} \\
0 & 0 & 0 & 1 & \beta^{T} \\
0 & 0 & 1 & 0 & \beta^{T} \\
\alpha-\beta & \alpha-\beta & \beta & \beta & A(G-u-v-x-y)
\end{array}\right)
$$

Again, by letting the $u$-th row (resp. $u$-th column) minus the $v$-th row (resp. $v$-th column) of $B$, and adding the $y$-th row (resp. $y$-th column) to the $v$-th row (resp. $v$-th column) of $B$, we obtain that $B$ is congruent to

$$
\left(\begin{array}{ccccc}
0 & 0 & 0 & 0 & \mathbf{0}^{T} \\
0 & 0 & 1 & 0 & \alpha^{T} \\
0 & 1 & 0 & 1 & \beta^{T} \\
0 & 0 & 1 & 0 & \beta^{T} \\
\mathbf{0} & \alpha & \beta & \beta & A(G-u-v-x-y)
\end{array}\right)=\left(\begin{array}{cc}
0 & \mathbf{0}^{T} \\
\mathbf{0} & A(G-u)
\end{array}\right)
$$

Thus $p(G)=p(G-u), n(G)=n(G-u)$ and $\eta(G)=\eta(G-u)+1$ by Lemma 2.4.

Remark 3.7. The Lemma 3.6 confirms that if a congruent vertex of III-type is deleted from a graph then the positive and negative inertia indices left unchanged, but the nullity reduces just one. Conversely, if we add a new vertex to a graph that consists of a congruent quadrangle with some other three vertices in this graph (briefly we refer to add a vertex of III-type in what follows) then the positive and negative inertia indices left unchanged, but the nullity adds just one. The graph transformation of deleting or adding vertices of III-type is called the (graph) transformation of III-type.

Remark 3.2, Remark 3.5 and Remark 3.7 provide us three transformations of graphs that keep the positive and negative inertia indices and change the nullity just one. By applying these transformations we will construct the graphs in $\mathcal{G}$. Let $\mathcal{G}_{1}$ be the set of connected graphs each of them is obtained from some $H \in \mathcal{H}$ by adding one vertex of I-type, $\mathcal{G}_{2}$ be the set of connected graphs each of them is obtained from some $H \in \mathcal{H}$ by adding one vertex of II-type and $\mathcal{G}_{3}$ be the set of connected graphs each of them is obtained from some $H \in \mathcal{H}$ by adding one vertex of III-type. At the end of this section, we would like to give an example to illustrate the constructions of the graphs in $\mathcal{G}_{i}(i=1,2,3)$.

Example 3.8. We know the path $P_{4}$, with spectrum $\operatorname{Spec}\left(P_{4}\right)=\{1.6180,0.6180$, $-0.6180,-1.6180\}$, is a graph belonging to $\mathcal{H}$. By adding a vertex $u$ of I-type to $P_{4}$ we obtain $H_{1} \in \mathcal{G}_{1}$ (see Figure 2) where $\operatorname{Spec}\left(H_{1}\right)=\{1.8478,0.7654,0,-0.7654,-1.8478\}$, 


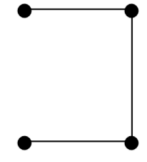

$P_{4}$

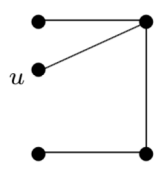

$H_{1}$

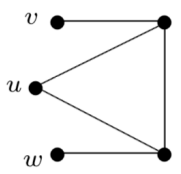

$\mathrm{H}_{2}$

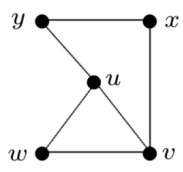

$H_{3}$

Figure 2: The graphs $P_{4}, H_{1}, H_{2}$ and $H_{3}$.

adding a vertex $u$ of II-type to $P_{4}$ we obtain $H_{2} \in \mathcal{G}_{2}$ where $\operatorname{Spec}\left(H_{2}\right)=\{2.3028,0.6180$, $0,-1.3028,-1.6180\}$. Finally, by adding a vertex $u$ of III-type to $P_{4}$ we obtain $H_{3} \in \mathcal{G}_{3}$, where $\operatorname{Spec}\left(H_{3}\right)=\{2.4812,0.6889,0,-1.1701,-2\}$. In fact, $u v$ and $x y$ is a pair of independent edges in $H_{3}$. Clearly, $N(u) \backslash\{v, y\}=N(v) \backslash\{u, x\}=\{w\}$ and $N(x) \backslash\{y, v\}=$ $N(y) \backslash\{x, u\}=\emptyset$. Thus $C_{4}=u v x y$ is a congruent quadrangle of $H_{3}$.

Clearly, $G=K_{1,2} \cup P_{2}$ is a non-connected graph in $\mathcal{G}$, and all such graphs we collect in $\mathcal{G}^{-}=\{G \in \mathcal{G} \mid G$ is disconnected $\}$. Additionally, $H_{1}$ and $H_{2}$ shown in Figure 2 are graphs with pendant vertex belonging to $\mathcal{G}$, and all such graphs we collect in $\mathcal{G}^{+}=\{G \in$ $\mathcal{G} \mid G$ is connected with a pendant vertex $\}$. In next section, we firstly determine the graphs in $\mathcal{G}^{-}$and $\mathcal{G}^{+}$.

\section{The characterization of graphs in $\mathcal{G}^{-}$and $\mathcal{G}^{+}$}

The following result completely characterizes the disconnected graphs of $\mathcal{G}$.

Theorem 4.1. Let $G$ be a graph of order $n \geq 5$. Then $G \in \mathcal{G}^{-}$if and only if $G \cong$ $K_{s}+K_{t}+K_{1}, H+K_{1}$ or $K_{s}+K_{n-s} \backslash$ e for $e \in E\left(K_{n-s}\right)$, where $H \in \mathcal{H}$ is connected and $s+t=n-1, s, t \geq 2$.

Proof. All the graphs displayed in Theorem 4.1 have two positive and one zero eigenvalues by simple observation. Now we prove the necessity.

Let $G \in \mathcal{G}^{-}$, and $H_{1}, H_{2}, \ldots, H_{k}(k \geq 2)$ the components of $G$. Since $\lambda_{1}\left(H_{i}\right) \geq 0$ for $i=1,2, \ldots, k$ and $\lambda_{4}(G)<0, G$ has two or three components and so $k \leq 3$.

First assume that $G=H_{1}+H_{2}+H_{3}$. It is easy to see that $G$ has exactly one isolated vertex due to $\eta(G)=1$ and $p(G)=2$. Without loss of generality, let $H_{3} \cong K_{1}$. Since $\lambda_{3}(G)=0$ and $\lambda_{1}\left(H_{i}\right)>0(i=1,2)$, we have $\lambda_{2}\left(H_{1}\right)<0$ and $\lambda_{2}\left(H_{2}\right)<0$. By Theorem 2.7 (2), $G \cong K_{s}+K_{t}+K_{1}$ as desired, where $s+t=n-1$ and $s, t \geq 2$.

Next assume that $G=H_{1}+H_{2}$. If $H_{1} \cong K_{1}$, then

$$
\begin{aligned}
\lambda_{1}(G)=\lambda_{1}\left(H_{2}\right) \geq \lambda_{2}(G) & =\lambda_{2}\left(H_{2}\right)>\lambda_{3}(G)= \\
0=\lambda_{1}\left(H_{1}\right) & >\lambda_{4}(G)=\lambda_{3}\left(H_{2}\right)<0 .
\end{aligned}
$$

Thus $H_{2} \cong H \in \mathcal{H}$, and so $G \cong H+K_{1}$ as desired. If $\left|H_{i}\right| \geq 2$ for $i=1,2$, then one of $\lambda_{2}\left(H_{1}\right)$ and $\lambda_{2}\left(H_{2}\right)$ is equal to zero and another is less than zero because $\lambda_{3}(G)=0$ and $\lambda_{4}(G)<0$. Without loss of generality, let $\lambda_{2}\left(H_{1}\right)<0$ and $\lambda_{2}\left(H_{2}\right)=0$. We have $\lambda_{3}\left(H_{1}\right) \leq \lambda_{2}\left(H_{1}\right)<0$, in addition, $\lambda_{3}\left(H_{2}\right)<0$ since $\eta(G)=1$. By Theorem 2.7 (2), $H_{1} \cong K_{s}$ for some $s \geq 2$ and by Theorem 2.7 (1), $H_{2} \cong K_{n-s} \backslash e$.

We complete this proof.

In terms of Theorem 2.6, we will determine all connected graphs with a pendant vertex satisfying $p(G)=2$ and $\eta(G)=d$ for any positive integer $d$. 
Theorem 4.2. Let $G$ be a connected graph of order $n$ with a pendant vertex. Then $p(G)=$ 2 and $\eta(G)=d \geq 1$ if and only if $G \cong K_{1, r}(u) \odot{ }^{k} K_{n_{1}, \ldots, n_{l}}$, where $r+n_{1}+n_{2}+\cdots+$ $n_{l}-(l+1)=d$.

Proof. Let $G=K_{1, r}(u) \odot^{k} K_{n_{1}, \ldots, n_{l}}$ and $v u$ is a pendant edge of $G$. By deleting $v$ and $u$ from $G$ we obtain $H=G-\{u, v\}=(r-1) K_{1} \cup K_{n_{1}, \ldots, n_{l}}$. It is well known that $p\left(K_{n_{1}, \ldots, n_{l}}\right)=1$ and $\eta\left(K_{n_{1}, \ldots, n_{l}}\right)=n_{1}+\cdots+n_{l}-l$. From Lemma 2.3 , we have

$$
\begin{aligned}
& p(G)=p(H)+1=p\left(K_{n_{1}, \ldots, n_{l}}\right)+1=2, \\
& \eta(G)=\eta(H)=(r-1)+\left(n_{1}+\cdots+n_{l}-l\right)=d .
\end{aligned}
$$

Conversely, let $G$ be a graph with a pendant vertex and $p(G)=2$. By Theorem 2.6, we have $G \cong K_{1, r}(u) \odot^{k} K_{n_{1}, \ldots, n_{l}}$. According to the arguments above, we know that $\eta(G)=r+n_{1}+n_{2}+\cdots+n_{l}-(l+1)=d$.

From Theorem 4.2, it immediately follows the result that completely characterizes the graphs in $\mathcal{G}^{+}$.

Corollary 4.3. A connected graph $G \in \mathcal{G}^{+}$if and only if $G \cong K_{1,2}(u) \odot^{k} K_{n-3}$ or $G \cong K_{1,1}(u) \odot^{k} K_{n-2} \backslash$ e for $e \in E\left(K_{n-2}\right)$.

Proof. By Theorem 4.2, we have $G \in \mathcal{G}^{+}$if and only if $G \cong K_{1, r}(u) \odot^{k} K_{n_{1}, \ldots, n_{l}}$, where $r+n_{1}+n_{2}+\cdots+n_{l}-(l+1)=1$ and $r, l, n_{1}, \ldots, n_{l} \geq 1$. It gives two solutions: one is $r=2, n_{1}=n_{2}=\cdots=n_{l}=1$ and $l=n-3$ which leads to $G \cong K_{1,2}(u) \odot^{k} K_{n-3}$; another is $r=1, n_{1}=2, n_{2}=\cdots=n_{l}=1$ and $l=n-2$ which leads to $G \cong K_{1,1}(u) \odot^{k} K_{n-2} \backslash e$ for $e \in E\left(K_{n-2}\right)$.

Let $\mathcal{G}^{*}$ denote the set of all connected graphs in $\mathcal{G}$ without pendant vertices. Then $\mathcal{G}=\mathcal{G}^{-} \cup \mathcal{G}^{+} \cup \mathcal{G}^{*}$. Therefore, in order to characterize $\mathcal{G}$, it remains to consider those graphs in $\mathcal{G}^{*}$.

\section{The characterization of graphs in $\mathcal{G}^{*}$}

First we introduce some symbols which will be persisted in this section. Let $G \in \mathcal{G}^{*}$. The eigenvalues of $G$ can be arranged as:

$$
\lambda_{1}(G) \geq \lambda_{2}(G)>\lambda_{3}(G)=0>\lambda_{4}(G) \geq \cdots \geq \lambda_{n}(G) .
$$

We choose $v^{*} \in V(G)$ such that $d_{G}\left(v^{*}\right)=\delta(G)=t$, and denote by $X=N_{G}\left(v^{*}\right)$ and $Y=V(G)-N_{G}\left[v^{*}\right]$. Then $t=|X| \geq 2$ since $G$ has no pendant vertices. In addition, $|Y|>0$ since otherwise $G$ would be a complete graph. First we characterize the induced subgraph $G[Y]$ in the following result.

Lemma 5.1. $G[Y] \cong K_{n-t-1} \backslash e, K_{1}+K_{n-t-2}$ or $K_{n-t-1}$.

Proof. First we suppose that $Y$ is an independent set. If $|Y| \geq 3$, then $\lambda_{4}(G) \geq$ $\lambda_{4}\left(G\left[Y \cup\left\{v^{*}\right\}\right]\right)=0$ by Theorem 2.1, a contradiction. Hence $|Y| \leq 2$, and so $G[Y] \cong K_{1}$ or $G[Y] \cong K_{2} \backslash e=2 K_{1}$.

Next we suppose that $G[Y]$ contains some edges. We distinguish the following three situations. 
If $\lambda_{2}(G[Y])>0$, we have $p(G[Y]) \geq 2$. For any $x \in X$, the induced subgraph $G\left[\left\{v^{*}, x\right\} \cup Y\right]$ has a pendant vertex $v^{*}$ by our assumption. By Lemma 2.2 and Lemma 2.3, we have $p(G) \geq p\left(G\left[\left\{v^{*}, x\right\} \cup Y\right]\right)=p(G[Y])+1 \geq 3$, a contradiction.

If $\lambda_{2}(G[Y])<0$, by Theorem 2.7 (2) we have $G[Y] \cong K_{n-t-1}$ as desired.

At last assume that $\lambda_{2}(G[Y])=0$. If $\lambda_{3}(G[Y])<0$, by Theorem 2.7 (1), we have $G[Y] \cong K_{n-t-1} \backslash e, K_{1}+K_{n-t-2}$ as desired. If $\lambda_{3}(G[Y])=0$, by Lemma 2.3 we have $p\left(G\left[\left\{v^{*}, x\right\} \cup Y\right]\right)=p(G[Y])+1=2$ and $\eta\left(G\left[\left\{v^{*}, x\right\} \cup Y\right]\right)=\eta(G[Y]) \geq 2$, which implies that $\lambda_{4}(G) \geq \lambda_{4}\left(G\left[\left\{v^{*}, x\right\} \cup Y\right]\right)=0$, a contradiction.

We complete this proof.

First assume that $Y=\left\{y_{1}\right\}$. If $G[X]=K_{t}$, then $G=K_{n} \backslash v^{*} y_{1}$. However $K_{n} \backslash$ $v^{*} y_{1} \notin \mathcal{G}^{*}$ since $p\left(K_{n} \backslash v^{*} y_{1}\right)=1$. Thus there exist $x_{1} \not x_{2}$ in $X$. Then $N_{G}\left(x_{1}\right)=$ $N_{G}\left(x_{2}\right)$ and $N_{G}\left(v^{*}\right)=N_{G}\left(y_{1}\right)$. It follows that $\eta(G) \geq 2$ by Lemma 3.1. Next assume that $Y=\left\{y_{1}, y\right\}$ is an independent set. We have $N_{G}\left(v^{*}\right)=N_{G}\left(y_{1}\right)=N_{G}(y)$ since $d_{G}\left(y_{1}\right), d_{G}(y) \geq d_{G}\left(v^{*}\right)=\delta(G)$. Thus, by Lemma 3.1 we have $\eta(G)=\eta\left(G-y_{1}\right)+1=$ $\eta\left(G-y_{1}-y\right)+2 \geq 2$. Thus we only need to consider the case that $G[Y]$ contains at least one edge. Concretely, we distinguish three situations in accordance with the proof of Lemma 5.1:

(a) $G[Y] \cong K_{n-t-2}+K_{1}$ in case of $\lambda_{2}(G[Y])=0$ and $\lambda_{3}(G[Y])<0$, where $n-t-2 \geq 2$

(b) $G[Y] \cong K_{n-t-1} \backslash e$ in case of $\lambda_{2}(G[Y])=0$ and $\lambda_{3}(G[Y])<0$, where $|Y|=$ $n-t-1 \geq 3$

(c) $G[Y] \cong K_{n-t-1}$ in case of $\lambda_{2}(G[Y])<0$, where $|Y|=n-t-1 \geq 2$.

In the following, we deal with situation (a) in Lemma 5.2, (b) in Lemma 5.3 and (c) in Lemma 5.4, 5.7 and Lemma 5.15. We will see that the graph $G \in \mathcal{G}^{*}$ illustrated in (a) and (b) can be constructed from some $H \in \mathcal{H}$ by the graph transformations of I-, II- and III-type, but (c) can not.

Lemma 5.2. If $G[Y] \cong K_{n-t-2}+K_{1}$, where $n-t-2 \geq 2$, then $G \in \mathcal{G}_{1}$.

Proof. Since $G[Y]$ is isomorphic to $K_{n-t-2}+K_{1}(n-t-2 \geq 2), Y$ exactly contains one isolated vertex of $G[Y]$, say $y$. We have $N_{G}\left(v^{*}\right)=N_{G}(y)$ and thus $y$ is a congruent vertex of I-type. By Lemma 3.1, we have $p(G)=p(G-y)$ and $\eta(G)=\eta(G-y)+1$. Notice that $G-y$ is connected, we have $G-y \in \mathcal{H}$, and so $G \in \mathcal{G}_{1}$. Such a graph $G$, displayed in Figure 3 (1), we call the $v^{*}$-graph of I-type.

In Figure 3 and Figure 5, two ellipses joining with one full line denote some edges between them. A vertex and an ellipse joining with one full line denote some edges between them, and with two full lines denote that this vertex joins all vertices in the ellipse. Two vertices join with same location of an ellipse denote that they have same neighbours in this ellipse.

It needs to mention that the $v^{*}$-graph of I-type characterized in Lemma 5.2, is a graph obtained from $H \in \mathcal{H}$ by adding a new vertex joining the neighbors of a minimum degree vertex of $H$.

For $S \subseteq V(G)$ and $u \in V(G)$, let $N_{S}(u)=N_{G}(u) \cap S$ and $N_{S}[u]=N_{G}[u] \cap S$.

Lemma 5.3. Let $G[Y] \cong K_{n-t-1} \backslash$ e, where $n-t-1 \geq 3$ and $e=y y^{\prime}$. Then $G \in \mathcal{G}_{1}$ if $N_{X}(y)=N_{X}\left(y^{\prime}\right)$ and $G \in \mathcal{G}_{2}$ otherwise. 


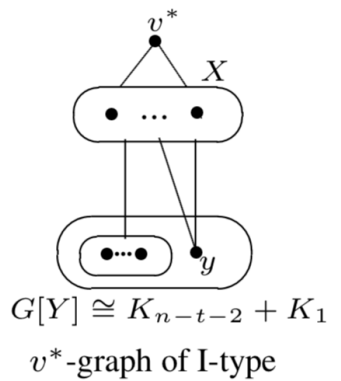

(1)

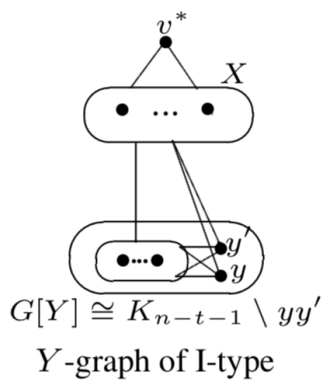

(2)

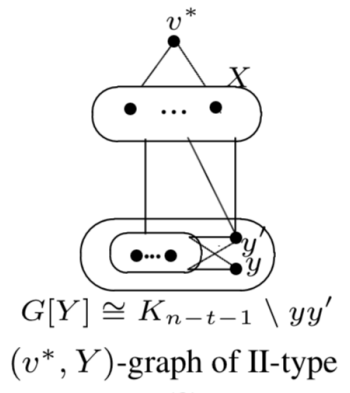

(3)

Figure 3: The structure of some graphs.

Proof. Since $n-t-1 \geq 3$, there is $y^{*} \in Y$ other than $y$ and $y^{\prime}$. It is clear that $N_{G}(y)=$ $N_{X}(y) \cup\left(Y \backslash\left\{y, y^{\prime}\right\}\right)$ and $N_{G}\left(y^{\prime}\right)=N_{X}\left(y^{\prime}\right) \cup\left(Y \backslash\left\{y, y^{\prime}\right\}\right)$, and thus $N_{G}(y)=N_{G}\left(y^{\prime}\right)$ if and only if $N_{X}(y)=N_{X}\left(y^{\prime}\right)$. We consider the following cases.

Case 1. $N_{X}(y)=N_{X}\left(y^{\prime}\right)$.

By assumption, $N_{G}(y)=N_{G}\left(y^{\prime}\right)$, thus $y$ and $y^{\prime}$ are congruent vertices of I-type. By Lemma 3.1, we have $p(G)=p(G-y)$ and $\eta(G)=\eta(G-y)+1$. Since $G-y$ is connected, we have $G-y \in \mathcal{H}$ and so $G \in \mathcal{G}_{1}$. Such a $G$, displayed in Figure 3 (2), we call the $Y$-graph of I-type.

Case 2. $N_{X}(y) \neq N_{X}\left(y^{\prime}\right)$.

First suppose that exactly one of $N_{X}(y)$ and $N_{X}\left(y^{\prime}\right)$ is empty, say $N_{X}(y)=\emptyset$ and $N_{X}\left(y^{\prime}\right) \neq \emptyset$. Then $y y^{*}$ is a pendant edge of the induced subgraph $G\left[X \cup\left\{y, y^{\prime}, y^{*}, v^{*}\right\}\right]$. By Lemma 2.2 and Lemma 2.3, we have

$$
2=p(G) \geq p\left(G\left[X \cup\left\{y, y^{\prime}, y^{*}, v^{*}\right\}\right]\right)=p\left(G\left[X \cup\left\{y^{\prime}, v^{*}\right\}\right]\right)+1 \geq 2 .
$$

Thus

$$
\begin{gathered}
p\left(G\left[X \cup\left\{y, y^{\prime}, y^{*}, v^{*}\right\}\right]\right)=2 \quad \text { and } \\
p\left(G\left[X \cup\left\{y^{\prime}, v^{*}\right\}\right]\right)=1 .
\end{gathered}
$$

We see that $\lambda_{2}\left(G\left[X \cup\left\{y^{\prime}, v^{*}\right\}\right]\right)=0$ (since otherwise $\lambda_{2}\left(G\left[X \cup\left\{y^{\prime}, v^{*}\right\}\right]\right)<0$ and then $G\left[X \cup\left\{y^{\prime}, v^{*}\right\}\right]$ is a complete graph, but $\left.y^{\prime} \nsim v^{*}\right)$. If $\lambda_{3}\left(G\left[X \cup\left\{y^{\prime}, v^{*}\right\}\right]\right)=0$, we have

$$
\eta\left(G\left[X \cup\left\{y, y^{\prime}, y^{*}, v^{*}\right\}\right]\right)=\eta\left(G\left[X \cup\left\{y^{\prime}, v^{*}\right\}\right]\right) \geq 2,
$$

which implies

$$
\lambda_{4}(G) \geq \lambda_{4}\left(G\left[X \cup\left\{y, y^{\prime}, y^{*}, v^{*}\right\}\right]\right)=0,
$$

a contradiction. If $\lambda_{3}\left(G\left[X \cup\left\{y^{\prime}, v^{*}\right\}\right]\right)<0$, then $G\left[X \cup\left\{y^{\prime}, v^{*}\right\}\right] \cong K_{t+2} \backslash e$ or $K_{t+1}+K_{1}$ by Theorem 2.7 (1). Notice that $G\left[X \cup\left\{y^{\prime}, v^{*}\right\}\right]$ is connected, we get $G\left[X \cup\left\{y^{\prime}, v^{*}\right\}\right] \cong$ $K_{t+2} \backslash e$ where $e=v^{*} y^{\prime}$. Thus $N_{X}\left(y^{\prime}\right)=X$ and so $N_{G}\left(y^{\prime}\right)=X \cup\left(Y \backslash\left\{y, y^{\prime}\right\}\right)=$ $N_{G}\left(v^{*}\right) \cup N_{G}(y)$ is a disjoint union. Additionally, $\left\{y^{\prime}, v^{*}, y\right\}$ is an independent set in $G$, we see that $y^{\prime}$ is a congruent vertex of II-type. Thus $p(G)=p\left(G-y^{\prime}\right)$ and $\eta(G)=$ 

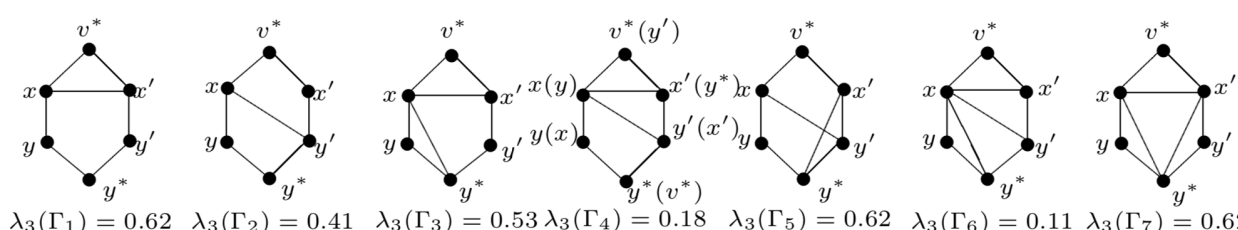

$\lambda_{3}\left(\Gamma_{1}\right)=0$.
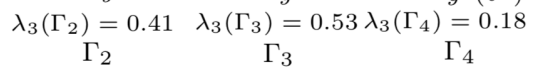

$\Gamma_{5}$
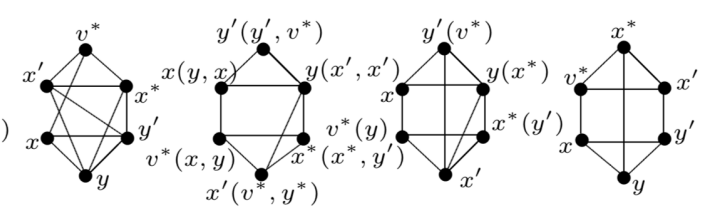

$\lambda_{3}\left(\Gamma_{8}\right)=0.28$

$\Gamma_{8}$

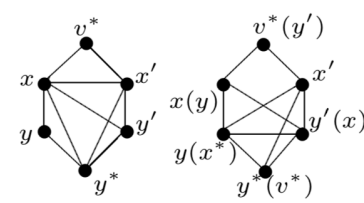

$\Gamma_{9}$

$\Gamma_{10}$

$\Gamma_{11}$

$\Gamma_{12}$

$\Gamma_{13}$

$\Gamma_{14}$
$\left.\Gamma_{14}\right)=0$

Figure 4: The graphs $\Gamma_{1}, \Gamma_{2}, \ldots, \Gamma_{14}$.

$\eta\left(G-y^{\prime}\right)+1$ by Lemma 3.4. This implies that $G-y^{\prime} \in \mathcal{H}$, and so $G \in \mathcal{G}_{2}$. Such a $G$, displayed in Figure 3 (3), we call the $\left(v^{*}, Y\right)$-graph of II-type.

Next suppose that $N_{X}(y), N_{X}\left(y^{\prime}\right) \neq \emptyset$, without loss of generality, assume that $N_{X}\left(y^{\prime}\right) \backslash N_{X}(y) \neq \emptyset$. Then there exists $x^{\prime} \in N_{X}\left(y^{\prime}\right) \backslash N_{X}(y)$. Thus $x^{\prime} \sim y^{\prime}$ and $x^{\prime} \nsim y$. Now by taking some $x \in N_{X}(y)$, we see that $C_{6}=v^{*} x y y^{*} y^{\prime} x^{\prime}$ is a 6 -cycle in $G$. Note that $x$ may joins each vertex in $\left\{x^{\prime}, y^{\prime}, y^{*}\right\}$ and $x^{\prime}$ may joins $y^{*}$. By distinguishing different situations in according with the number of edges we have

$$
G\left[v^{*}, x, y, y^{*}, y^{\prime}, x^{\prime}\right] \cong \begin{cases}C_{6} & \text { no edge; } \\ \Gamma_{1} \text { or } \Gamma_{2} & \text { one edges; } \\ \Gamma_{3}, \Gamma_{4} \text { or } \Gamma_{5} & \text { two edges; } \\ \Gamma_{6}, \Gamma_{7} \text { or } \Gamma_{8} & \text { three edges; } \\ \Gamma_{9} & \text { four edges. }\end{cases}
$$

However $C_{6}$ and $\Gamma_{1}, \ldots, \Gamma_{8}$ and $\Gamma_{9}$ are all forbidden subgraphs of $G$ (see Figure 4).

We complete this proof.

It remains to characterize the graph $G \in \mathcal{G}^{*}$ satisfying $G[Y] \cong K_{n-t-1}$. Such a graph $G$ we call $X$-complete if $G[X]$ is also complete graph, and $X$-imcomplete otherwise. The following result characterizes the $X$-imcomplete graphs.

Lemma 5.4. Let $G[Y] \cong K_{n-t-1}$, where $n-t-1 \geq 2$, and $G$ is $X$-imcomplete. Then $G \in \mathcal{G}_{1}$ if there exist two non-adjacent vertices $x_{1} \nsim x_{2}$ in $G[X]$ such that $N_{Y}\left(x_{1}\right)=$ $N_{Y}\left(x_{2}\right)$ and $G \in \mathcal{G}_{3}$ otherwise.

Proof. Let $X=\left\{x_{1}, x_{2}, \ldots, x_{t}\right\}$ and $Y=\left\{y_{1}, y_{2}, \ldots, y_{n-t-1}\right\}$. Then $V(G)=\left\{v^{*}\right\} \cup$ $X \cup Y$ and $Y$ induces $K_{n-t-1}$. Let $x$ and $x^{\prime}$ be two non-adjacent vertices in $X$. Since $d_{G}(x) \geq d_{G}\left(v^{*}\right)$ and $n-t-1 \geq 2$, we have $\left|N_{Y}(x)\right| \geq 1$ and $|Y| \geq 2$, respectively. First we give some claims.

Claim 5.5. If $x \ngtr x^{\prime}$ in $G[X]$ then one of $N_{Y}(x)$ and $N_{Y}\left(x^{\prime}\right)$ includes another. If $N_{Y}(x) \subset N_{Y}\left(x^{\prime}\right)$ then $\left|N_{Y}(x)\right|=1$ and $N_{Y}\left(x^{\prime}\right)=Y$. 
Proof. On the contrary, let $y \in N_{Y}(x) \backslash N_{Y}\left(x^{\prime}\right)$ and $y^{\prime} \in N_{Y}\left(x^{\prime}\right) \backslash N_{Y}(x)$, then $G\left[v^{*}, x, x^{\prime}, y, y^{\prime}\right] \cong C_{5}$. Thus one of $N_{Y}(x)$ and $N_{Y}\left(x^{\prime}\right)$ includes another. Now assume that $N_{Y}(x) \subset N_{Y}\left(x^{\prime}\right)$. If $\left|N_{Y}(x)\right| \geq 2$, say $\left\{y, y^{\prime}\right\} \subseteq N_{Y}(x)$, then $x^{\prime} \sim y, y^{\prime}$ and exists $y^{*} \in N_{Y}\left(x^{\prime}\right) \backslash N_{Y}(x)$. Thus $G\left[v^{*}, x, x^{\prime}, y, y^{\prime}, y^{*}\right] \cong \Gamma_{10}$ (see Figure 4). However $p\left(\Gamma_{10}\right)=3$. Hence $\left|N_{Y}(x)\right|=1$, and we may assume that $N_{Y}(x)=\{y\}$. If $N_{Y}\left(x^{\prime}\right) \neq Y$, then there exists $y^{\prime} \in Y \backslash N_{Y}\left(x^{\prime}\right)$. Also, there exists $y^{*} \in N_{Y}\left(x^{\prime}\right) \backslash N_{Y}(x)$. We have $G\left[v^{*}, x, x^{\prime}, y, y^{\prime}, y^{*}\right] \cong \Gamma_{4}$ (see the labels in the parentheses of Figure 4), but $p\left(\Gamma_{4}\right)=3$. Thus $N_{Y}\left(x^{\prime}\right)=Y$.

Claim 5.6. If $x \neq x^{\prime}$ in $G[X]$ then $N_{X}(x)=N_{X}\left(x^{\prime}\right)$.

Proof. On the contrary, we may assume that $x^{*} \in N_{X}\left(x^{\prime}\right) \backslash N_{X}(x)$. Then $x^{*} \sim x^{\prime}$ and $x^{*} \nsim x$, thus $\left|N_{Y}(x)\right| \geq 2$ since $\left|N_{G}(x)\right| \geq t$. By Claim 5.5, we have $N_{Y}\left(x^{*}\right), N_{Y}\left(x^{\prime}\right) \subseteq$ $N_{Y}(x)$. Then either $N_{Y}\left(x^{*}\right)=N_{Y}\left(x^{\prime}\right)=N_{Y}(x)$ or one of $N_{Y}\left(x^{*}\right)$ and $N_{Y}\left(x^{\prime}\right)$ is a proper subset of $N_{Y}(x)$ (without loss of generality, assume that $N_{Y}\left(x^{*}\right) \subset N_{Y}(x)$, and then $\left|N_{Y}\left(x^{*}\right)\right|=1$ and $N_{Y}(x)=Y$ by Claim 5.5).

Suppose that $N_{Y}(x)=N_{Y}\left(x^{*}\right)=N_{Y}\left(x^{\prime}\right)$. Take $y, y^{\prime} \in N_{Y}(x)$, we see that $G\left[v^{*}, x, x^{*}, x^{\prime}, y, y^{\prime}\right] \cong \Gamma_{11}$ (see Figure 4). However $p\left(\Gamma_{11}\right)=3$.

Suppose that $\left|N_{Y}\left(x^{*}\right)\right|=1$ and $N_{Y}(x)=Y$. Let $N_{Y}\left(x^{*}\right)=\{y\}$ and there exists another $y^{\prime} \in Y$. Then $G\left[v^{*}, x, x^{*}, x^{\prime}, y, y^{\prime}\right]$ is isomorphic $\Gamma_{13}$ (see Figure 4) if $x^{\prime} \sim y, y^{\prime}$, or isomorphic to $\Gamma_{12}$ (see Figure 4) if $x^{\prime} \sim y$ and $x^{\prime} \nsim y^{\prime}$, or isomorphic to $\Gamma_{14}$ (see Figure 4) if $x^{\prime} \not y$ and $x^{\prime} \sim y^{\prime}$. However $p\left(\Gamma_{12}\right)=p\left(\Gamma_{13}\right)=3$ and $\lambda_{4}\left(\Gamma_{14}\right)=0$. We are done.

Now we distinguish the following cases to prove our result.

Case 1. There exist $x_{1} \not x_{2}$ such that $N_{Y}\left(x_{1}\right)=N_{Y}\left(x_{2}\right)$.

Since $x_{1} \not x_{2}$, we have $N_{X}\left(x_{1}\right)=N_{X}\left(x_{2}\right)$ by Claim 5.6, so $N_{G}\left(x_{1}\right)=N_{G}\left(x_{2}\right)$. Thus $x_{1}$ and $x_{2}$ are congruent vertices of I-type. By Lemma 3.1, $p(G)=p\left(G-x_{1}\right)$ and $\eta(G)=\eta\left(G-x_{1}\right)+1$. Thus $G-x_{1} \in \mathcal{H}$ and so $G \in \mathcal{G}_{1}$. Such a $G$, displayed in Figure 5 (1), we call the $X$-graph of I-type.

Case 2. For each pair of $x \neq x^{\prime} \in X, N_{Y}(x) \neq N_{Y}\left(x^{\prime}\right)$.

By Claim 5.5, without loss of generality, assume that $N_{Y}(x) \subset N_{Y}\left(x^{\prime}\right)$ and then $N_{Y}(x)=\{y\}$ and $N_{Y}\left(x^{\prime}\right)=Y$. Thus $y \sim x, x^{\prime}$ and furthermore we will show that $X \subseteq$ $N_{G}(y)$. In fact, let $x^{*} \in X \backslash\left\{x, x^{\prime}\right\}$ (if any), if $x \not x^{*}$, we have $N_{Y}\left(x^{*}\right) \supseteq N_{Y}(x)=\{y\}$ by Claim 5.6. Thus $y \sim x^{*}$. Otherwise, $x \sim x^{*}$ and thus $x^{\prime} \sim x^{*}$ since $N_{X}(x)=N_{X}\left(x^{\prime}\right)$ by Claim 5.6. Now take $y^{\prime} \in Y \backslash\{y\}$. If $y \not x^{*}$, then $G\left[v^{*}, x, x^{\prime}, x^{*}, y, y^{\prime}\right]$ is isomorphic to $\Gamma_{12}$ (see the first labels in the parentheses of Figure 4) while $x^{*} \nsim y^{\prime}$, or isomorphic to $\Gamma_{13}$ (see the labels in the parentheses of Figure 4) while $x^{*} \sim y^{\prime}$, but $p\left(\Gamma_{12}\right)=p\left(\Gamma_{13}\right)=3$. It follows that $N_{G}(y)=X \cup(Y \backslash\{y\})$ since $Y$ induces a clique.

On the other hand, since $d_{G}(x) \geq|X|=t, x \neq x^{\prime}$ and $N_{Y}(x)=\{y\}$, we have $N_{X}(x)=X \backslash\left\{x, x^{\prime}\right\}$ and so $N_{X}\left(\overline{x^{\prime}}\right)=X \backslash\left\{x, x^{\prime}\right\}$ by Claim 5.6. Thus $N_{G}(x)=$ $\left(X \backslash\left\{x, x^{\prime}\right\}\right) \cup\left\{v^{*}, y\right\}$ and $N_{G}\left(x^{\prime}\right)=\left(X \backslash\left\{x, x^{\prime}\right\}\right) \cup Y \cup\left\{v^{*}\right\}$. Hence the quadrangle $C_{4}=x v^{*} x^{\prime} y$ is congruent, where $x v^{*}$ and $x^{\prime} y$ is a pair of congruent edges of $C_{4}$. It gives that $x, v^{*}, x^{\prime}, y$ are congruent vertices of III-type. By Lemma 3.6, we have $p(G)=$ $p(G-x)$ and $\eta(G)=\eta(G-x)+1$ thus $G-x \in \mathcal{H}$, and so $G \in \mathcal{G}_{3}$. Such a $G$, displayed in Figure 5 (2), we call the $\left(v^{*}, X, Y\right)$-graph of III-type.

We complete this proof. 


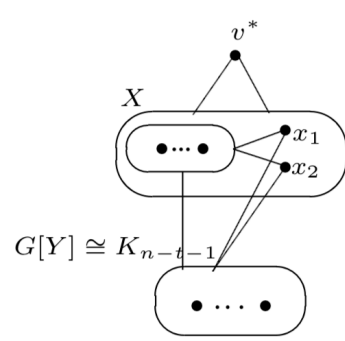

$X$-graph of I-type

(1)

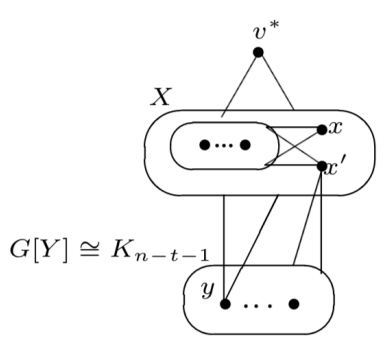

$\left(v^{*}, X, Y\right)$-graph of III-type

(2)

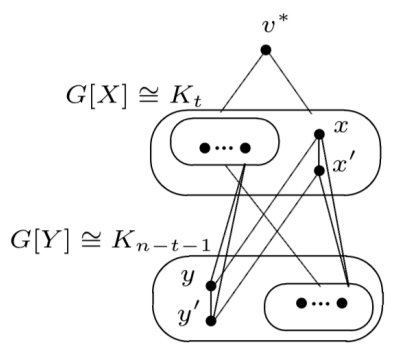

$(X, Y)$-graph of III-type

(3)

Figure 5: The structure of some graphs.

At last we focus on characterizing $X$-complete graph $G \in \mathcal{G}^{*}$, i.e., $G[X] \cong K_{t}$ and $G[Y] \cong K_{n-t-1}$. A $X$-complete graph $G \in \mathcal{G}^{*}$ is called reduced if one of $N_{Y}\left(x_{i}\right)$ and $N_{Y}\left(x_{j}\right)$ is a subset of another for any $x_{i} \neq x_{j} \in X$ and non-reduced otherwise. Thus the $X$-complete graphs are partitioned into a disjoint union of the reduced and non-reduced $X$-complete graphs. Concretely, for a reduced $X$-complete graph $G \in \mathcal{G}^{*}$, we may assume that $\emptyset=N_{Y}\left(v^{*}\right) \subseteq N_{Y}\left(x_{1}\right) \subseteq N_{Y}\left(x_{2}\right) \subseteq \cdots \subseteq N_{Y}\left(x_{t}\right)$; for a non-reduced $(X, Y)$ complete graph $G \in \mathcal{G}^{*}$, there exist some $x \neq x^{\prime} \in X$ such that $N_{Y}(x) \backslash N_{Y}\left(x^{\prime}\right) \neq \emptyset$ and $N_{Y}\left(x^{\prime}\right) \backslash N_{Y}(x) \neq \emptyset$. Such vertices $x$ and $x^{\prime}$ are called non-reduced vertices. It remains to characterize the reduced and non-reduced $X$-complete graphs in what follows.

Lemma 5.7. Let $G \in \mathcal{G}^{*}$ be a non-reduced $X$-complete graph and $x, x^{\prime}$ be non-reduced vertices. Then $G \in \mathcal{G}_{3}$.

Proof. Since $x, x^{\prime}$ are non-reduced vertices, there exist $y \in N_{Y}(x) \backslash N_{Y}\left(x^{\prime}\right)$ and $y^{\prime} \in$ $N_{Y}\left(x^{\prime}\right) \backslash N_{Y}(x)$. Then $x, x^{\prime}, y^{\prime}, y$ induces $C_{4}$ (see Figure 5 (3)). It suffices to verify that $C_{4}$ is congruent. Clearly, $N_{G}(x) \supset(X \backslash\{x\}) \cup\left\{v^{*}\right\}$ and $N_{G}\left(x^{\prime}\right) \supset\left(X \backslash\left\{x^{\prime}\right\}\right) \cup\left\{v^{*}\right\}$. If there exists $y^{*} \in N_{Y}(x) \backslash N_{Y}\left(x^{\prime}\right)$ other than $y$, then $G\left[v^{*}, x, x^{\prime}, y^{\prime}, y, y^{*}\right] \cong \Gamma_{12}$ (see the second labels in the parentheses of Figure 4), however $\Gamma_{12}$ is a forbidden subgraph of $G$. Hence $N_{Y}(x) \backslash N_{Y}\left(x^{\prime}\right)=\{y\}$. Similarly, $N_{Y}\left(x^{\prime}\right) \backslash N_{Y}(x)=\left\{y^{\prime}\right\}$. On the other aspect, $x \in N_{X}(y) \backslash N_{X}\left(y^{\prime}\right)$ and $x^{\prime} \in N_{X}\left(y^{\prime}\right) \backslash N_{X}(y)$. If there exists $x^{*} \in N_{X}(y) \backslash N_{X}\left(y^{\prime}\right)$ other than $x$, then $G\left[v^{*}, x, x^{\prime}, x^{*}, y, y^{\prime}\right] \cong \Gamma_{10}$ (see the labels in the parentheses of Figure 4), however $\Gamma_{10}$ is a forbidden subgraph of $G$. Hence $N_{X}(y) \backslash N_{X}\left(y^{\prime}\right)=\{x\}$. Similarly, $N_{X}\left(y^{\prime}\right) \backslash N_{X}(y)=\left\{x^{\prime}\right\}$. Hence $N_{X}(y) \backslash\{x\}=N_{X}\left(y^{\prime}\right) \backslash\left\{x^{\prime}\right\}$. Note that $N_{G}(y) \supset Y \backslash\{y\}$ and $N_{G}\left(y^{\prime}\right) \supset Y \backslash\left\{y^{\prime}\right\}$, we have $N_{G}(y) \backslash\left\{y^{\prime}, x\right\}=\left(Y \backslash\left\{y, y^{\prime}\right\}\right) \cup\left(N_{X}(y) \backslash\{x\}\right)=$ $N_{G}\left(y^{\prime}\right) \backslash\left\{x^{\prime}, y\right\}$. Hence the quadrangle $C_{4}=x x^{\prime} y^{\prime} y$ is congruent, where $x x^{\prime}$ and $y^{\prime} y$ is a pair of congruent edges. It follows that $x, x^{\prime}, y^{\prime}, y$ are congruent vertices of III-type. By Lemma 3.6, we have $p(G)=p(G-x)$ and $\eta(G)=\eta(G-x)+1$. Thus $G-x \in \mathcal{H}$, and so $G \in \mathcal{G}_{3}$. Such a $G$, displayed in Figure 5 (3), we call the $(X, Y)$-graph of III-type.

We complete this proof.

To characterize the reduced $X$-complete graph, we need the notion of canonical graph which is introduced in [7]. For a graph $G$, a relation $\rho$ on $V(G)$ we mean that $u \rho v$ iff $u \sim v$ and $N_{G}(u) \backslash v=N_{G}(v) \backslash u$. Clearly, $\rho$ is symmetric and transitive. In accordance with $\rho$, 
the vertex set is decomposed into classes:

$$
V(G)=V_{1} \cup V_{2} \cup \cdots \cup V_{k},
$$

where $v_{i} \in V_{i}$ and $V_{i}=\left\{x \in V(G) \mid x \rho v_{i}\right\}$. By definition of $\rho, V_{i}$ induces a clique $K_{n_{i}}$ where $n_{1}+n_{2}+\cdots+n_{k}=n=|V(G)|$, and vertices of $V_{i}$ join that of $V_{j}$ iff $v_{i} \sim v_{j}$ in $G$. We call the induced subgraph $G\left[\left\{v_{1}, v_{2}, \ldots, v_{k}\right\}\right]$ as the canonical graph of $G$, denoted by $G_{c}$. Thus $G=G_{c}\left[K_{n_{1}}, K_{n_{2}}, \ldots, K_{n_{k}}\right]$ is a generalized lexicographic product of $G_{c}$ (by $K_{n_{1}}, K_{n_{2}}, \ldots, K_{n_{k}}$ ).

Let $G$ be a reduced $X$-complete graph. From (5.1) we have $G=G_{c}\left[K_{n_{1}}, K_{n_{2}}, \ldots\right.$, $\left.K_{n_{k}}\right]$, where $G_{c}=G\left[\left\{v_{1}, v_{2}, \ldots, v_{k}\right\}\right]$ and $V_{i}=\left\{x \in V(G) \mid x \rho v_{i}\right\}$ induces clique $K_{n_{i}}$. Without loss of generality, assume $v_{1}=v^{*}$. Let $X_{c}=N_{G_{c}}\left(v_{1}\right)$ and $Y_{c}=$ $\left\{v_{2}, v_{3}, \ldots, v_{k}\right\} \backslash X_{c}$. Clearly, $G_{c}\left[X_{c}\right]$ is a clique since $X_{c}$ is a subset of $X$ and $X$ induces a clique in $G$. Furthermore, $G_{c}\left[Y_{c}\right]$ is a clique since $Y_{c}$ is a subset of $Y$ and $Y$ induces a clique in $G$. Thus $G_{c}$ is also a $X_{c}$-complete graph. Additionally, since $G$ is reduced, $G_{c}$ is also reduced. Let $t_{c}=d_{G_{c}}\left(v_{1}\right)$ and $X_{c}=\left\{x_{1}, x_{2}, \ldots, x_{t_{c}}\right\}, Y_{c}=$ $\left\{y_{1}, y_{2}, \ldots, y_{k-t_{c}-1}\right\}$. We may assume $N_{Y_{c}}\left(v_{1}\right) \subset N_{Y_{c}}\left(x_{1}\right) \subset \cdots \subset N_{Y_{c}}\left(x_{t_{c}}\right)$ and $N_{X_{c}}\left(y_{1}\right) \subset \cdots \subset N_{X_{c}}\left(y_{k-t_{c}-1}\right)$. Therefore,

$$
0=\left|N_{Y_{c}}\left(v_{1}\right)\right|<\left|N_{Y_{c}}\left(x_{1}\right)\right|<\cdots<\left|N_{Y_{c}}\left(x_{t_{c}}\right)\right| \leq\left|Y_{c}\right|=k-t_{c}-1,
$$

and

$$
0 \leq\left|N_{X_{c}}\left(y_{1}\right)\right|<\left|N_{X_{c}}\left(y_{2}\right)\right|<\cdots<\left|N_{X_{c}}\left(y_{k-t_{c}-1}\right)\right| \leq\left|X_{c}\right|=t_{c}
$$

From Equation (5.2), we have $t_{c} \leq k-t_{c}-1$. Similarly, $k-t_{c}-2 \leq t_{c}$ from Equation (5.3). Thus $k-2 \leq 2 t_{c} \leq k-1$, and so $t_{c}=\left\lceil\frac{k}{2}\right\rceil-1$.

If $k$ is even, then $t_{c}=\frac{k}{2}-1$. From Equation (5.2), we have $\left|N_{Y_{c}}\left(x_{i}\right)\right|=i$ for $i=1,2, \ldots, t_{c}$. Thus we may assume that

$$
\begin{aligned}
N_{Y_{c}}\left(v_{1}\right) & =\emptyset, \\
N_{Y_{c}}\left(x_{1}\right) & =\left\{y_{\frac{k}{2}}\right\}, \\
& \vdots \\
N_{Y_{c}}\left(x_{\frac{n}{2}-2}\right) & =\left\{y_{\frac{k}{2}}, \ldots, y_{3}\right\}, \\
N_{Y_{c}}\left(x_{\frac{n}{2}-1}\right) & =\left\{y_{\frac{k}{2}}, \ldots, y_{2}\right\} .
\end{aligned}
$$

This implies that $G \cong G_{k}$ where $G_{k}$ is defined in Section 2. Similarly, $G \cong G_{k}$ if $k$ is odd. Thus we obtain the following result.

Lemma 5.8. Let $G$ be a reduced $X$-complete graph. Then $G_{c} \cong G_{k}$ where $k \geq 2$ is determined in (5.1).

Let $G \in \mathcal{G}^{*}$ be a reduced $X$-complete graph. The following lemma gives a characterization for $G$. First we cite a result due to Oboudi in [5].

Lemma 5.9 ([5]). Let $G=G_{3}\left[K_{n_{1}}, K_{n_{2}}, K_{n_{3}}\right]$, where $n_{1}, n_{2}, n_{3}$ are some positive integers. Then the following hold:

(1) If $n_{1}=n_{2}=n_{3}=1$, that is $G \cong P_{3}$, then $\lambda_{3}(G)=-\sqrt{2}$;

(2) If $n_{1}=n_{2}=1$ and $n_{3} \geq 2$, then $\lambda_{3}(G)=-1$; 
(3) If $n_{1} n_{2}>1$, then $\lambda_{3}(G)=-1$.

We know that any graph $G$ is a generalized lexicographic product of its canonical graph, i.e., $G=G_{c}\left[K_{n_{1}}, K_{n_{2}}, \ldots, K_{n_{k}}\right]$. We also have $G_{c}=G_{k}$ if $G$ is reduced $X$-complete by Lemma 5.8. Furthermore, the following result prove that $4 \leq k \leq 13$.

Lemma 5.10. Let $G \in \mathcal{G}^{*}$ be a reduced $X$-complete graph. Then there exists $4 \leq k \leq 13$ such that $G=G_{k}\left[K_{n_{1}}, K_{n_{2}}, \ldots, K_{n_{k}}\right]$.

Proof. By Lemma 5.8, $G=G_{k}\left[K_{n_{1}}, K_{n_{2}}, \ldots, K_{n_{k}}\right]$ for some $k$. If $k=1$ or 2 then $G \cong K_{n} \notin \mathcal{G}^{*}$, and so $k \geq 3$. If $k=3$, then $G=G_{3}\left[K_{n_{1}}, K_{n_{2}}, K_{n_{3}}\right]$. Thus $\lambda_{3}(G)<0$ by Lemma 5.9, a contradiction. Hence $k \geq 4$. On the other hand, since $G_{c}=G_{k}$ is an induced subgraph of $G$, we have $\lambda_{4}\left(G_{k}\right) \leq \lambda_{4}(G)<0$ by Theorem 2.1. Note that $G_{14}$ is an induced subgraph of $G_{k}$ (by Remark 2.8) for $k \geq 15$, we have $\lambda_{4}\left(G_{k}\right) \geq \lambda_{4}\left(G_{14}\right)=0$. It implies that $k \leq 13$.

Next we consider the converse of Lemma 5.10. In other words, we will try to find the values of $n_{1}, \ldots, n_{k}$ such that $p\left(G_{k}\left[K_{n_{1}}, \ldots, K_{n_{k}}\right]\right)=2$ and $\eta\left(G_{k}\left[K_{n_{1}}, \ldots, K_{n_{k}}\right]\right)=1$, where $4 \leq k \leq 13$ and $n=n_{1}+n_{2}+\cdots+n_{k}$. For the simplicity, we use notation in [8] to denote

$$
\begin{aligned}
G_{2 s}\left[K_{n_{1}}, \ldots, K_{n_{2 s}}\right] & =B_{2 s}\left(n_{1}, \ldots, n_{s} ; n_{s+1}, \ldots, n_{2 s}\right) \quad \text { and } \\
G_{2 s+1}\left[K_{n_{1}}, \ldots, K_{n_{2 s+1}}\right] & =B_{2 s+1}\left(n_{1}, \ldots, n_{s} ; n_{s+1}, \ldots, n_{2 s} ; n_{2 s+1}\right) .
\end{aligned}
$$

By Remark 3.2 in [6], we know

$$
\begin{aligned}
H_{0} & =B_{2 s}\left(n_{1}, \ldots, n_{s} ; n_{s+1}, \ldots, n_{2 s}\right) \\
& \cong B_{2 s}\left(n_{s+1} \ldots, n_{2 s} ; n_{1}, \ldots, n_{s}\right)=H_{0}^{\prime} \quad \text { and } \\
H_{1} & =B_{2 s+1}\left(n_{1}, \ldots, n_{s} ; n_{s+1}, \ldots, n_{2 s} ; n_{2 s+1}\right) \\
& \cong B_{2 s+1}\left(n_{s+1}, \ldots, n_{2 s} ; n_{1}, \ldots, n_{s} ; n_{2 s+1}\right)=H_{1}^{\prime} .
\end{aligned}
$$

In what follows, we always take $H_{0}$ and $H_{1}$, in which $\left(n_{1}, \ldots, n_{s}\right)$ is prior to $\left(n_{s+1}, \ldots\right.$, $\left.n_{2 s}\right)$ in dictionary ordering, instead of $H_{0}^{\prime}$ and $H_{1}^{\prime}$. For example we use $B_{6}(4,3,2 ; 4,3,1)$ instead of $B_{6}(4,3,1 ; 4,3,2)$ and $B_{7}(5,3,2 ; 5,2,4 ; 8)$ instead of $B_{7}(5,2,4 ; 5,3,2 ; 8)$.

For $4 \leq k \leq 13$, let

$$
\mathcal{B}_{k}(n)=\left\{G=B_{k}\left(n_{1}, \ldots, n_{k}\right) \mid n=n_{1}+\cdots+n_{k}, n_{i} \geq 1\right\} .
$$

Let $\mathcal{B}_{k}^{+}(n), \mathcal{B}_{k}^{00}(n), \mathcal{B}_{k}^{0}(n)$ and $\mathcal{B}_{k}^{-}(n)$ denote the set of graphs in $\mathcal{B}_{k}(n)$ satisfying $\lambda_{3}(G)>$ 0 for $G \in \mathcal{B}_{k}^{+}(n), \lambda_{4}(G)=\lambda_{3}(G)=0$ for $G \in \mathcal{B}_{k}^{00}(n), \lambda_{4}(G)<\lambda_{3}(G)=0$ for $G \in \mathcal{B}_{k}^{0}(n)$ and $\lambda_{3}(G)<0$ for $G \in \mathcal{B}_{k}^{-}(n)$, respectively. Clearly, $\mathcal{B}_{k}(n)=\mathcal{B}_{k}^{+}(n) \cup$ $\mathcal{B}_{k}^{00}(n) \cup \mathcal{B}_{k}^{0}(n) \cup \mathcal{B}_{k}^{-}(n)$ is disjoint union and $G=G_{k}\left[K_{n_{1}}, K_{n_{2}}, \ldots, K_{n_{k}}\right] \in \mathcal{B}_{k}^{0}(n)$ if $G \in \mathcal{G}^{*}$ is a reduced $X$-complete graph by Lemma 5.10. In what follows, we further show that $n \leq 13$. First, one can verify the following result by using computer.

Lemma 5.11. $\mathcal{B}_{k}^{0}(14)=\emptyset$ for $4 \leq k \leq 13$ (it means that there are no reduced $X$-complete graphs of order 14).

Proof. For $4 \leq k \leq 13$, the $k$-partition of 14 gives a solution $\left(n_{1}, n_{2}, \ldots, n_{k}\right)$ of the equation $n_{1}+n_{2}+\cdots+n_{k}=14$ that corresponds a graph $G=B_{k}\left(n_{1}, n_{2}, \ldots, n_{k}\right) \in$ $\mathcal{B}_{k}(14)$. By using computer, we exhaust all the graphs of $\mathcal{B}_{k}(14)$ to find that there is no any graph $G \in \mathcal{B}_{k}(14)$ with $\lambda_{4}(G)<\lambda_{3}(G)=0$. It implies that $\mathcal{B}_{k}^{0}(14)=\emptyset$. 
In [6], Oboudi gave all the integers $n_{1}, \ldots, n_{k}$ satisfying $\lambda_{2}\left(B_{k}\left(n_{1}, \ldots, n_{k}\right)\right)>0$ and $\lambda_{3}\left(B_{k}\left(n_{1}, \ldots, n_{k}\right)\right)<0$ for $4 \leq k \leq 9$. For simplicity, we only cite this result for $k=5$ and the others are listed in Appendix B.

Theorem 5.12 ([6]). Let $G=B_{5}\left(n_{1}, n_{2} ; n_{3}, n_{4} ; n_{5}\right)$, where $n_{1}, n_{2}, n_{3}, n_{4}, n_{5}$ are some positive integers. Then $\lambda_{2}(G)>0$ and $\lambda_{3}(G)<0$ if and only if $G$ is isomorphic to one of the following graphs:
(1) $B_{5}(a, w ; 1,1 ; 1)$;
(6) $B_{5}(a, 1 ; x, w ; 1)$;
(11) $B_{5}(x, w ; 1, d ; 1)$;
(2) $B_{5}(a, x ; 1, d ; 1)$;
(7) $B_{5}(a, 1 ; x, y ; e)$;
(12) $B_{5}(x, w ; 1,1 ; e)$;
(3) $B_{5}(a, x ; 1, y ; z)$;
(8) $B_{5}(a, 1 ; 1, d ; e)$;
(13) $B_{5}(1, b ; 1, d ; 1)$;
(4) $B_{5}(a, x ; 1,1 ; e)$;
(9) $B_{5}(w, x ; y, 1 ; e)$;
(14) $B_{5}(1, b ; 1, x ; y)$;
(5) $B_{5}(a, 1 ; c, 1 ; e)$;
(10) $B_{5}(x, b ; 1,1 ; 1)$;
(15) $B_{5}(1, x ; 1, y ; e)$;

(16) 63 specific graphs: 13 graphs of order 10, 25 graphs of order 11, and 25 graphs of order 12 ,

where $a, b, c, d, e, x, y, z, w$ are some positive integers such that $x \leq 2, y \leq 2, z \leq 2$ and $w \leq 3$.

Lemma 5.13. Let $G \in \mathcal{B}_{k}(n)$, where $4 \leq k \leq 9$ and $n \geq 14$. If $G \notin \mathcal{B}_{k}^{-}(n)$, then $G$ has an induced subgraph $\Gamma \in \mathcal{B}_{k}(14) \backslash \mathcal{B}_{k}^{-}(14)$.

Proof. We prove this lemma by induction on $n$. If $n=14$, since $G \in \mathcal{B}_{k}(14) \backslash \mathcal{B}_{k}^{-}$(14), our result is obviously true by taking $\Gamma=G$. Let $n \geq 15$ and $G^{\prime} \in \mathcal{B}_{k}(n-1)$ be an induced subgraph of $G$. If $G^{\prime} \notin \mathcal{B}_{k}^{-}(n-1)$, then $G^{\prime}$ has an induced subgraph $\Gamma \in \mathcal{B}_{k}(14) \backslash \mathcal{B}_{k}^{-}(14)$ by induction hypothesis, and so does $G$. Hence it suffices to prove that $G$ contains an induced subgraph $G^{\prime} \in \mathcal{B}_{k}(n-1) \backslash \mathcal{B}_{k}^{-}(n-1)$ for $n \geq 15$ in the following. We will prove that there exists $G^{\prime} \in \mathcal{B}_{5}(n-1) \backslash \mathcal{B}_{5}^{-}(n-1)$ for $n \geq 15$, and it can be similarly proved for the other $k$ which we keep in the Appendix B.

Let $G=B_{5}\left(n_{1}, n_{2} ; n_{3}, n_{4} ; n_{5}\right) \in \mathcal{B}_{5}(n)$. Then one of

$$
\begin{array}{ll}
H_{1}=B_{5}\left(n_{1}-1, n_{2} ; n_{3}, n_{4} ; n_{5}\right), & H_{2}=B_{5}\left(n_{1}, n_{2}-1 ; n_{3}, n_{4} ; n_{5}\right), \\
H_{3}=B_{5}\left(n_{1}, n_{2} ; n_{3}-1, n_{4} ; n_{5}\right), & H_{4}=B_{5}\left(n_{1}, n_{2} ; n_{3}, n_{4}-1 ; n_{5}\right) \text { and } \\
H_{5}=B_{5}\left(n_{1}, n_{2} ; n_{3}, n_{4} ; n_{5}-1\right) &
\end{array}
$$

must belong to $\mathcal{B}_{5}(n-1)$. On the contrary, assume that $H_{i} \in \mathcal{B}_{5}^{-}(n-1)$ for $i=1,2, \ldots, 5$. Then $H_{i}$ is a graph belonging to (1) - (15) in Theorem 5.12 since $\left|H_{i}\right|=n-1 \geq 14$.

First we consider $H_{1}$. If $H_{1}$ is a graph belonging to (1) of Theorem 5.12, then $H_{1}=$ $B_{5}(a, w ; 1,1 ; 1)$ where $n_{1}-1=a, n_{2}=w, n_{3}=n_{4}=n_{5}=1$, and hence $G=B_{5}(a+1$, $w ; 1,1 ; 1) \in \mathcal{B}_{5}^{-}(n)$, a contradiction. Similarly, $H_{1}$ cannot belong to (2)-(8) of Theorem 5.12. If $H_{1}$ is a graph belonging to (9) of Theorem 5.12, then $H_{1}=B_{5}(w, x ; y, 1 ; e)$ where $n_{1}-1=w, n_{2}=x, n_{3}=y, n_{4}=1, n_{5}=e$. Since $w \leq 3$, we have $n_{1} \leq 4$. If $n_{1}<4$ then $w+1 \leq 3$ and $G=B_{5}(w+1, x ; y, 1 ; e) \in \mathcal{B}_{5}^{-}(n)$, a contradiction. Now assume that $n_{1}=4$. Then $H_{1}=B_{5}(3, x ; y, 1 ; e)$. Since $x, y \in\{1,2\}$, we have $G \in\left\{B_{5}(4,1 ; 1,1 ; e), B_{5}(4,2 ; 1,1 ; e), B_{5}(4,1 ; 2,1 ; e), B_{5}(4,2 ; 2,1 ; e)\right\}$. However $B_{5}(4,1 ; 1,1 ; e), B_{5}(4,2 ; 1,1 ; e), B_{5}(4,1 ; 2,1 ; e)$ belong to (4), (5) of Theorem 5.12 which contradicts our assumption. Thus $G=B_{5}(4,2 ; 2,1 ; e)$. By Theorem $5.12, G=$ 
$B_{5}(4,2 ; 2,1 ; e) \notin \mathcal{B}_{5}^{-}(n)$, and also its induced subgraph $B_{5}(4,2 ; 2,1 ; e-1) \notin \mathcal{B}_{5}^{-}(n-1)$, a contradiction. Hence $H_{1}$ belongs to (10) - (15) of Theorem 5.12, from which we see that $n_{1}-1$ is either $x$ or 1 . Thus $n_{1} \leq 3$ due to $x \leq 2$.

By the same method, we can verify that $n_{2} \leq 3$ if $H_{2} \in \mathcal{B}_{5}^{-}(n-1) ; n_{3} \leq 3$ if $H_{3} \in \mathcal{B}_{5}^{-}(n-1) ; n_{4} \leq 3$ if $H_{4} \in \mathcal{B}_{5}^{-}(n-1)$ and $n_{5} \leq 2$ if $H_{5} \in \mathcal{B}_{5}^{-}(n-1)$. Hence $n=n_{1}+\cdots+n_{5} \leq 14$, a contradiction. We are done.

Lemma 5.14 ([6]). If $n \geq 14$, then $\mathcal{B}_{k}^{-}(n)=\emptyset$ for $10 \leq k \leq 13$.

Lemma 5.15. Given $4 \leq k \leq 13, \mathcal{B}_{k}^{0}(n)=\emptyset$ for $n \geq 14$ (it means that there are no reduced $X$-complete graphs of order $n \geq 14$ ).

Proof. Let $G \in \mathcal{B}_{k}^{0}(n)$ and $n \geq 14$. Then $\lambda_{4}(G)<\lambda_{3}(G)=0$. First we assume that $4 \leq k \leq 9$. Since $G \notin \mathcal{B}_{k}^{-}(n), G$ has an induced subgraphs $\Gamma \in \mathcal{B}_{k}(14) \backslash \mathcal{B}_{k}^{-}(14)$ by Lemma 5.13. Thus $\lambda_{3}(\Gamma) \geq 0$. Furthermore, we have $\lambda_{3}(\Gamma)=0$ since otherwise $0<\lambda_{3}(\Gamma) \leq \lambda_{3}(G)$. Additionally, $\lambda_{4}(\Gamma) \leq \lambda_{4}(G)<0$, we have $\Gamma \in \mathcal{B}_{k}^{0}(14)$, contrary to Lemma 5.11. Next we assume that $10 \leq k \leq 13$. By deleting $n-14$ vertices from $G$, we may obtain an induced subgraph $\Gamma \in \mathcal{B}_{k}(14)$. By Lemma 5.14, we have $\lambda_{3}(\Gamma) \geq 0$, and then $\lambda_{3}(\Gamma)=0$ by the arguments above. Additionally, $\lambda_{4}(\Gamma) \leq \lambda_{4}(G)<0$, we have $\Gamma \in \mathcal{B}_{k}^{0}(14)$ which also contradicts Lemma 5.11.

By Lemma 5.15, we know that, for any reduced $X$-complete graph $G \in \mathcal{G}^{*}$, there exists $4 \leq k \leq 13$ and $n \leq 13$ such that $G \in \mathcal{B}_{k}^{0}(n)$. Let

$$
\mathcal{B}^{*}=\left\{G=B_{k}\left(n_{1}, n_{2}, \ldots, n_{k}\right) \in \mathcal{B}_{k}^{0}(n) \mid 4 \leq k \leq 13 \text { and } n \leq 13\right\} .
$$

Thus $G \in \mathcal{G}^{*}$ is a reduced $X$-complete graph if and only if $G \in \mathcal{B}^{*}$.

Remark 5.16. Clearly, $\mathcal{B}=\cup_{4 \leq k \leq 13, n \leq 13} \mathcal{B}_{k}(n)$ contains finite graphs. By using computer we can exhaust all the graphs of $\mathcal{B}$ to find out the graphs in $\mathcal{B}^{*}$. We list them in Table 1.

Recall that $\mathcal{G}_{1}, \mathcal{G}_{2}$ and $\mathcal{G}_{3}$ are the set of connected graphs each of them is obtained from some $H \in \mathcal{H}$ by adding one vertex of I, II, III-type, respectively. Summarizing Lemmas 5.2, 5.3, 5.4, 5.15 and Theorem 4.2, finally we give the characterization of the connected graphs in $\mathcal{G}$.

Theorem 5.17. Let $G$ be a connected graph of order $n \geq 5$. Then $G \in \mathcal{G}$ if and only if $G$ is isomorphic to one of the following graphs listed in (1), (2) and (3):

(1) $K_{1,2}(u) \odot^{k} K_{n-3}$ or $K_{1,1}(u) \odot^{k} K_{n-2} \backslash$ efor $e \in E\left(K_{n-2}\right)$;

(2) the graphs belonging to $\mathcal{G}_{1}, \mathcal{G}_{2}$ or $\mathcal{G}_{3}$;

(3) the 802 specific graphs belonging to $\mathcal{B}^{*}$ some of which we list in Table 1.

If $G^{*}$ is obtained from $G \in \mathcal{G}$ by adding one vertex of I, II or III-type, then the positive and negative indices of $G^{*}$ left unchanged, but the nullity adds just one. Repeating this process, we can get a class of graphs which has two positive eigenvalues and $s$ zero eigenvalues, where $s \geq 2$ is any integer. However, by using the I, II and III-type (graph) transformations, we can not get all such graphs. For example, $H=B_{10}(1,1,2,3,2 ; 1,1,1,1,1)$ is a graph satisfying $p(H)=2$ and $\eta(H)=2$ that can not be constructed by above (graph) transformation. Hence the characterization of graphs with $p(H)=2$ and $\eta(H)=s$ (especially $\eta(H)=2$ ) is also an attractive problem. 
Table 1: All graphs of $\mathcal{B}^{*}$.

\begin{tabular}{|c|c|c|}
\hline$k$ & $\mathcal{B}^{*}$ & Number \\
\hline 4 & $\begin{array}{l}B_{4}(3,2 ; 3,2) ; B_{4}(4,3 ; 2,2), B_{4}(4,3 ; 3,1) ; B_{4}(5,4 ; 2,1), B_{4}(5,2 ; 2,3), \\
B_{4}(3,4 ; 2,3), B_{4}(4,1 ; 3,4), B_{4}(5,2 ; 4,1) ; B_{4}(7,3 ; 2,1), B_{4}(4,6 ; 2,1), \\
B_{4}(7,2 ; 2,2), B_{4}(3,6 ; 2,2), B_{4}(4,2 ; 2,5), B_{4}(3,3 ; 2,5), B_{4}(7,2 ; 3,1), \\
B_{4}(3,6 ; 3,1), B_{4}(6,1 ; 3,3), B_{4}(6,1 ; 4,2) .\end{array}$ & 18 \\
\hline 5 & $\begin{array}{l}B_{5}(2,2 ; 2,2 ; 1) ; B_{5}(2,3 ; 1,2 ; 2), B_{5}(3,3 ; 2,1 ; 1) ; B_{5}(3,4 ; 1,1 ; 2), \\
B_{5}(3,4 ; 1,2 ; 1), B_{5}(1,3 ; 1,3 ; 3), B_{5}(2,2 ; 1,3 ; 3), B_{5}(2,4 ; 2,1 ; 2), \\
B_{5}(4,2 ; 3,1 ; 1) ; B_{5}(4,5 ; 1,1 ; 1), B_{5}(2,5 ; 1,1 ; 3), B_{5}(4,3 ; 1,1 ; 3), \\
B_{5}(1,4 ; 1,2 ; 4), B_{5}(3,2 ; 1,2 ; 4), B_{5}(2,5 ; 1,3 ; 1), B_{5}(4,3 ; 1,3 ; 1), \\
B_{5}(1,4 ; 1,4 ; 2), B_{5}(3,2 ; 1,4 ; 2), B_{5}(5,2 ; 2,1 ; 2), B_{5}(3,1 ; 2,3 ; 3), \\
B_{5}(3,1 ; 2,5 ; 1), B_{5}(4,1 ; 3,2 ; 2) ; B_{5}(3,7 ; 1,1 ; 1), B_{5}(6,4 ; 1,1 ; 1), \\
B_{5}(2,7 ; 1,1 ; 2), B_{5}(6,3 ; 1,1 ; 2), B_{5}(2,4 ; 1,1 ; 5), B_{5}(3,3 ; 1,1 ; 5), \\
B_{5}(2,7 ; 1,2 ; 1), B_{5}(6,3 ; 1,2 ; 1), B_{5}(1,6 ; 1,2 ; 3), B_{5}(5,2 ; 1,2 ; 3), \\
B_{5}(1,3 ; 1,2 ; 6), B_{5}(2,2 ; 1,2 ; 6), B_{5}(1,6 ; 1,3 ; 2), B_{5}(5,2 ; 1,3 ; 2), \\
B_{5}(2,4 ; 1,5 ; 1), B_{5}(3,3 ; 1,5 ; 1), B_{5}(2,2 ; 1,6 ; 2), B_{5}(2,7 ; 2,1 ; 1), \\
B_{5}(7,2 ; 2,1 ; 1), B_{5}(4,2 ; 2,1 ; 4), B_{5}(2,3 ; 2,1 ; 5), B_{5}(5,1 ; 2,3 ; 2), \\
B_{5}(5,1 ; 2,4 ; 1), B_{5}(3,2 ; 3,1 ; 4), B_{5}(6,1 ; 3,2 ; 1) .\end{array}$ & 47 \\
\hline 6 & See Table 2 of Appendix A & 138 \\
\hline 7 & See Table 3 of Appendix A & 161 \\
\hline 8 & See Table 4 of Appendix A & 205 \\
\hline 9 & See Table 5 of Appendix A & 124 \\
\hline 10 & See Table 6 of Appendix A & 78 \\
\hline 11 & $\begin{array}{l}B_{11}(1,1,1,2,1 ; 1,1,1,1,1 ; 1), B_{11}(2,1,1,1,1 ; 1,1,1,1,1 ; 1) \\
B_{11}(1,1,1,1,3 ; 1,1,1,1,1 ; 1), B_{11}(1,1,1,2,2 ; 1,1,1,1,1 ; 1), \\
B_{11}(1,1,2,1,2 ; 1,1,1,1,1 ; 1), B_{11}(1,1,2,2,1 ; 1,1,1,1,1 ; 1), \\
B_{11}(1,1,3,1,1 ; 1,1,1,1,1 ; 1), B_{11}(1,2,1,1,2 ; 1,1,1,1,1 ; 1), \\
B_{11}(1,2,2,1,1 ; 1,1,1,1,1 ; 1), B_{11}(1,3,1,1,1 ; 1,1,1,1,1 ; 1), \\
B_{11}(2,1,1,1,2 ; 1,1,1,1,1 ; 1), B_{11}(2,2,1,1,1 ; 1,1,1,1,1 ; 1), \\
B_{11}(1,1,1,1,2 ; 1,1,1,1,1 ; 2), B_{11}(1,1,2,1,1 ; 1,1,1,1,1 ; 2), \\
B_{11}(1,2,1,1,1 ; 1,1,1,1,1 ; 2), B_{11}(1,1,1,1,1 ; 1,1,1,1,1 ; 3), \\
B_{11}(1,1,1,1,2 ; 1,1,1,1,2 ; 1), B_{11}(1,1,1,2,1 ; 1,1,1,1,2 ; 1), \\
B_{11}(1,1,2,1,1 ; 1,1,1,1,2 ; 1), B_{11}(1,2,1,1,1 ; 1,1,1,1,2 ; 1), \\
B_{11}(1,1,2,1,1 ; 1,1,2,1,1 ; 1), B_{11}(1,2,1,1,1 ; 1,1,2,1,1 ; 1), \\
B_{11}(2,1,1,1,1 ; 1,1,2,1,1 ; 1), B_{11}(1,2,1,1,1 ; 1,2,1,1,1 ; 1) .\end{array}$ & 24 \\
\hline 12 & $\begin{array}{l}B_{12}(1,1,1,1,1,2 ; 1,1,1,1,1,1), B_{12}(1,1,1,1,2,1 ; 1,1,1,1,1,1), \\
B_{12}(1,1,1,2,1,1 ; 1,1,1,1,1,1), B_{12}(1,1,2,1,1,1 ; 1,1,1,1,1,1), \\
B_{12}(1,2,1,1,1,1 ; 1,1,1,1,1,1), B_{12}(2,1,1,1,1,1 ; 1,1,1,1,1,1) .\end{array}$ & 6 \\
\hline 13 & $B_{13}(1,1,1,1,1,1 ; 1,1,1,1,1,1 ; 1)$. & \\
\hline
\end{tabular}

\section{References}

[1] D. M. Cvetković, M. Doob and H. Sachs, Spectra of Graphs: Theory and Application, volume 87 of Pure and Applied Mathematics, Academic Press, New York, 1980.

[2] C. Godsil and G. Royle, Algebraic Graph Theory, volume 207 of Graduate Texts in Mathemat- 
ics, Springer-Verlag, New York, 2001, doi:10.1007/978-1-4613-0163-9.

[3] H. Ma, W. Yang and S. Li, Positive and negative inertia index of a graph, Linear Algebra Appl. 438 (2013), 331-341, doi:10.1016/j.laa.2012.07.014.

[4] M. R. Oboudi, Bipartite graphs with at most six non-zero eigenvalues, Ars Math. Contemp. 11 (2016), 315-325, doi:10.26493/1855-3974.749.264.

[5] M. R. Oboudi, On the third largest eigenvalue of graphs, Linear Algebra Appl. 503 (2016), 164-179, doi:10.1016/j.laa.2016.03.037.

[6] M. R. Oboudi, Characterization of graphs with exactly two non-negative eigenvalues, Ars Math. Contemp. 12 (2017), 271-286, doi:10.26493/1855-3974.1077.5b6.

[7] M. Petrović, On graphs with exactly one eigenvalue less than -1, J. Comb. Theory Ser. B 52 (1991), 102-112, doi:10.1016/0095-8956(91)90096-3.

[8] M. Petrović, Graphs with a small number of nonnegative eigenvalues, Graphs Combin. 15 (1999), 221-232, doi:10.1007/s003730050042.

[9] J. H. Smith, Symmetry and multiple eigenvalues of graphs, Glasnik Mat. Ser. III 12 (1977), 3-8, https: / / books.google.com/books? id=I1nZvGaOcscC\&pg=PA3.

[10] A. Torgašev, On graphs with a fixed number of negative eigenvalues, Discrete Math. 57 (1985), 311-317, doi:10.1016/0012-365x(85)90184-0.

[11] G. Yu, L. Feng and H. Qu, Signed graphs with small positive index of inertia, Electron. J. Linear Algebra 31 (2016), 232-243, doi:10.13001/1081-3810.1976. 


\section{Appendix A Five tables}

Appendix A contains 5 tables, in which there are 706 specific graphs: 4 graphs of order 10 , 32 graphs of order 11, 150 graphs of order 12, and 520 graphs of order 13 .

Table 2: $k=6$.

\begin{tabular}{|c|c|}
\hline$n$ & \\
\hline 10 & $\overline{B_{6}(1,2,2 ; 1,2,2), B_{6}(2,2,1 ; 1,2,2)}$; \\
\hline 11 & $\begin{array}{l}B_{6}(1,3,3 ; 1,1,2), B_{6}(2,3,2 ; 1,1,2), B_{6}(3,3,1 ; 1,1,2), B_{6}(1,3,3 ; 1,2,1), \\
B_{6}(2,3,2 ; 1,2,1), B_{6}(3,3,1 ; 1,2,1), B_{6}(2,1,1 ; 1,3,3), B_{6}(3,2,1 ; 2,1,2), \\
B_{6}(2,2,2 ; 2,2,1), B_{6}(3,1,2 ; 3,1,1)\end{array}$ \\
\hline 12 & $\begin{array}{l}B_{6}(1,4,4 ; 1,1,1), B_{6}(2,4,3 ; 1,1,1), B_{6}(3,4,2 ; 1,1,1), B_{6}(4,4,1 ; 1,1,1), \\
B_{6}(1,2,4 ; 1,1,3), B_{6}(1,4,2 ; 1,1,3), B_{6}(2,2,3 ; 1,1,3), B_{6}(2,4,1 ; 1,1,3), \\
B_{6}(3,2,2 ; 1,1,3), B_{6}(4,2,1 ; 1,1,3), B_{6}(1,3,1 ; 1,2,4), B_{6}(2,1,2 ; 1,2,4), \\
B_{6}(3,1,1 ; 1,2,4), B_{6}(1,4,2 ; 1,3,1), B_{6}(2,2,3 ; 1,3,1), B_{6}(2,4,1 ; 1,3,1), \\
B_{6}(3,2,2 ; 1,3,1), B_{6}(4,2,1 ; 1,3,1), B_{6}(2,1,2 ; 1,4,2), B_{6}(3,1,1 ; 1,4,2), \\
B_{6}(2,3,3 ; 2,1,1), B_{6}(4,1,3 ; 2,1,1), B_{6}(4,3,1 ; 2,1,1), B_{6}(2,3,2 ; 2,1,2), \\
B_{6}(3,2,2 ; 2,1,2), B_{6}(4,1,2 ; 2,1,2), B_{6}(2,3,1 ; 2,1,3), B_{6}(4,1,1 ; 2,1,3), \\
B_{6}(3,1,3 ; 2,2,1), B_{6}(3,2,2 ; 2,2,1), B_{6}(3,3,1 ; 2,2,1), B_{6}(3,1,1 ; 2,2,3), \\
B_{6}(2,3,1 ; 2,3,1), B_{6}(3,2,2 ; 3,1,1), B_{6}(4,2,1 ; 3,1,1), B_{6}(4,1,1 ; 4,1,1) ;\end{array}$ \\
\hline 13 & $\begin{array}{l}B_{6}(1,3,6 ; 1,1,1), B_{6}(1,6,3 ; 1,1,1), B_{6}(2,3,5 ; 1,1,1), B_{6}(2,6,2 ; 1,1,1), \\
B_{6}(3,3,4 ; 1,1,1), B_{6}(3,6,1 ; 1,1,1), B_{6}(4,3,3 ; 1,1,1), B_{6}(5,3,2 ; 1,1,1), \\
B_{6}(6,3,1 ; 1,1,1), B_{6}(1,2,6 ; 1,1,2), B_{6}(1,6,2 ; 1,1,2), B_{6}(2,2,5 ; 1,1,2), \\
B_{6}(2,6,1 ; 1,1,2), B_{6}(3,2,4 ; 1,1,2), B_{6}(4,2,3 ; 1,1,2), B_{6}(5,2,2 ; 1,1,2), \\
B_{6}(6,2,1 ; 1,1,2), B_{6}(1,2,3 ; 1,1,5), B_{6}(1,3,2 ; 1,1,5), B_{6}(2,2,2 ; 1,1,5), \\
B_{6}(2,2,5 ; 1,2,1), B_{6}(2,6,1 ; 1,2,1), B_{6}(3,2,4 ; 1,2,1), B_{6}(4,2,3 ; 1,2,1), \\
B_{6}(5,2,2 ; 1,2,1), B_{6}(2,3,1 ; 1,1,5), B_{6}(3,2,1 ; 1,1,5), B_{6}(1,2,6 ; 1,2,1), \\
B_{6}(1,6,2 ; 1,2,1), B_{6}(6,2,1 ; 1,2,1), B_{6}(1,5,1 ; 1,2,3), B_{6}(2,1,4 ; 1,2,3), \\
B_{6}(3,1,3 ; 1,2,3), B_{6}(4,1,2 ; 1,2,3), B_{6}(5,1,1 ; 1,2,3), B_{6}(2,1,1 ; 1,2,6), \\
B_{6}(1,5,1 ; 1,3,2), B_{6}(2,1,4 ; 1,3,2), B_{6}(3,1,3 ; 1,3,2), B_{6}(4,1,2 ; 1,3,2), \\
B_{6}(5,1,1 ; 1,3,2), B_{6}(2,2,2 ; 1,5,1), B_{6}(2,3,1 ; 1,5,1), B_{6}(3,2,1 ; 1,5,1), \\
B_{6}(2,1,1 ; 1,6,2), B_{6}(2,2,5 ; 2,1,1), B_{6}(2,5,2 ; 2,1,1), B_{6}(3,1,5 ; 2,1,1), \\
B_{6}(3,2,4 ; 2,1,1), B_{6}(3,3,3 ; 2,1,1), B_{6}(3,4,2 ; 2,1,1), B_{6}(3,5,1 ; 2,1,1), \\
B_{6}(4,2,3 ; 2,1,1), B_{6}(4,3,2 ; 2,1,1), B_{6}(5,2,2 ; 2,1,1), B_{6}(6,1,2 ; 2,1,1), \\
B_{6}(6,2,1 ; 2,1,1), B_{6}(2,2,4 ; 2,1,2), B_{6}(2,5,1 ; 2,1,2), B_{6}(3,1,4 ; 2,1,2), \\
B_{6}(3,2,3 ; 2,1,2), B_{6}(6,1,1 ; 2,1,2), B_{6}(2,2,3 ; 2,1,3), B_{6}(3,1,3 ; 2,1,3), \\
B_{6}(2,2,2 ; 2,1,4), B_{6}(3,1,2 ; 2,1,4), B_{6}(2,2,1 ; 2,1,5), B_{6}(3,1,1 ; 2,1,5), \\
B_{6}(2,5,1 ; 2,2,1), B_{6}(4,2,2 ; 2,2,1), B_{6}(5,1,2 ; 2,2,1), B_{6}(5,2,1 ; 2,2,1), \\
B_{6}(3,1,3 ; 2,2,2), B_{6}(4,1,2 ; 2,2,2), B_{6}(5,1,1 ; 2,2,2), B_{6}(3,1,2 ; 2,2,3), \\
B_{6}(4,1,2 ; 2,3,1), B_{6}(4,2,1 ; 2,3,1), B_{6}(3,1,2 ; 2,3,2), B_{6}(4,1,1 ; 2,3,2), \\
B_{6}(3,1,2 ; 2,4,1), B_{6}(3,2,1 ; 2,4,1), B_{6}(3,1,1 ; 2,4,2), B_{6}(3,3,2 ; 3,1,1), \\
B_{6}(3,4,1 ; 3,1,1), B_{6}(6,1,1 ; 3,1,1), B_{6}(3,3,1 ; 3,2,1), B_{6}(4,2,1 ; 3,2,1), \\
B_{6}(5,1,1 ; 3,2,1), B_{6}(4,1,1 ; 3,3,1) .\end{array}$ \\
\hline
\end{tabular}


Table 3: $k=7$.

\begin{tabular}{|c|c|}
\hline$n$ & \\
\hline 10 & $B_{7}(2,2,1 ; 1,1,2 ; 1), B_{7}(2,1,2 ; 2,1,1 ; 1)$ \\
\hline 11 & $\begin{array}{l}B_{7}(3,3,1 ; 1,1,1 ; 1), B_{7}(2,1,3 ; 1,1,1 ; 2), B_{7}(2,2,2 ; 1,1,2 ; 1), B_{7}(2,1,2 ; 1,1,2 ; 2), \\
B_{7}(1,2,1 ; 1,1,3 ; 2), B_{7}(2,1,1 ; 1,1,3 ; 2), B_{7}(1,2,3 ; 1,2,1 ; 1), B_{7}(1,2,2 ; 1,2,2 ; 1), \\
B_{7}(2,1,1 ; 1,2,3 ; 1), B_{7}(2,2,2 ; 2,1,1 ; 1), B_{7}(3,2,1 ; 2,1,1 ; 1), B_{7}(3,1,1 ; 3,1,1 ; 1)\end{array}$ \\
\hline 12 & $\begin{array}{l}B_{7}(1,3,4 ; 1,1,1 ; 1), B_{7}(3,1,4 ; 1,1,1 ; 1), B_{7}(3,3,2 ; 1,1,1 ; 1), B_{7}(1,2,4 ; 1,1,1 ; 2), \\
B_{7}(2,2,3 ; 1,1,1 ; 2), B_{7}(2,4,1 ; 1,1,1 ; 2), B_{7}(3,2,2 ; 1,1,1 ; 2), B_{7}(4,2,1 ; 1,1,1 ; 2), \\
B_{7}(1,1,4 ; 1,1,1 ; 3), B_{7}(3,1,2 ; 1,1,1 ; 3), B_{7}(1,3,3 ; 1,1,2 ; 1), B_{7}(2,2,3 ; 1,1,2 ; 1), \\
B_{7}(3,1,3 ; 1,1,2 ; 1), B_{7}(1,1,3 ; 1,1,2 ; 3), B_{7}(1,3,1 ; 1,1,2 ; 3), B_{7}(3,1,1 ; 1,1,2 ; 3), \\
B_{7}(1,3,2 ; 1,1,3 ; 1), B_{7}(3,1,2 ; 1,1,3 ; 1), B_{7}(1,2,2 ; 1,1,3 ; 2), B_{7}(1,3,1 ; 1,1,4 ; 1), \\
B_{7}(3,1,1 ; 1,1,4 ; 1), B_{7}(2,1,4 ; 1,2,1 ; 1), B_{7}(2,2,3 ; 1,2,1 ; 1), B_{7}(2,3,2 ; 1,2,1 ; 1), \\
B_{7}(4,2,1 ; 1,2,1 ; 1), B_{7}(2,1,2 ; 1,2,1 ; 3), B_{7}(1,2,2 ; 1,2,2 ; 2), B_{7}(1,3,1 ; 1,2,2 ; 2), \\
B_{7}(2,4,1 ; 1,2,1 ; 1), B_{7}(2,1,2 ; 1,2,2 ; 2), B_{7}(3,1,1 ; 1,2,2 ; 2), B_{7}(2,1,2 ; 1,2,3 ; 1), \\
B_{7}(1,3,2 ; 1,3,1 ; 1), B_{7}(3,1,1 ; 1,3,2 ; 1), B_{7}(2,3,2 ; 2,1,1 ; 1), B_{7}(2,3,1 ; 2,1,1 ; 2), \\
B_{7}(4,1,1 ; 2,1,1 ; 2), B_{7}(3,2,1 ; 2,2,1 ; 1), B_{7}(3,1,1 ; 2,2,1 ; 2) ;\end{array}$ \\
\hline 13 & 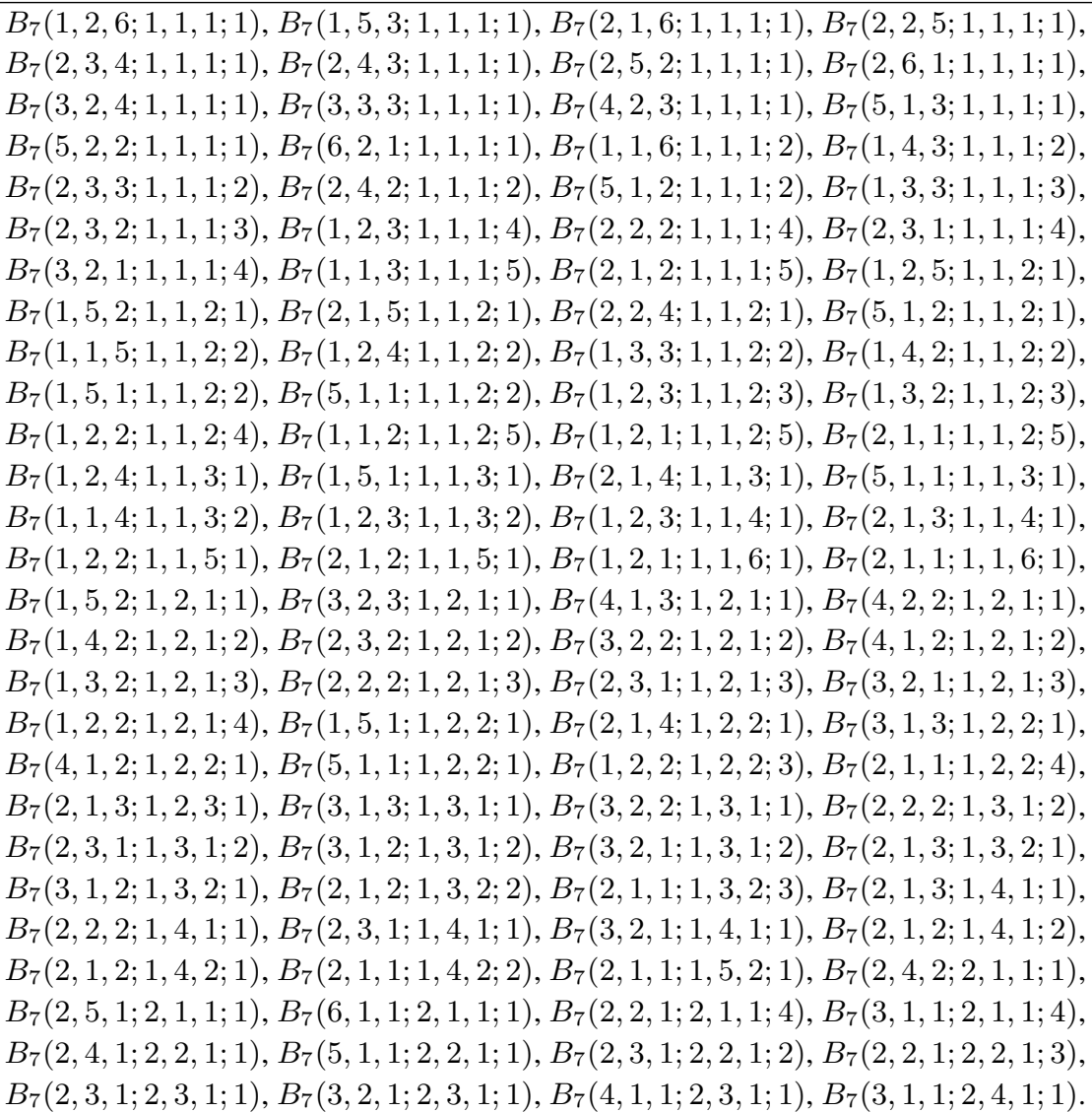 \\
\hline
\end{tabular}


Table 4: $k=8$.

\begin{tabular}{|c|c|}
\hline$n$ & $3^{*}$ \\
\hline 11 & $\begin{array}{l}B_{8}(1,2,1,2 ; 1,1,1,2), B_{8}(2,2,1,1 ; 1,1,1,2), B_{8}(1,2,2,1 ; 1,1,2,1), \\
B_{8}(1,2,1,1 ; 1,1,2,2), B_{8}(2,1,2,1 ; 1,2,1,1), B_{8}(2,1,1,1 ; 1,2,1,2) ;\end{array}$ \\
\hline 12 & $\begin{array}{l}B_{8}(1,1,3,3 ; 1,1,1,1), B_{8}(1,3,1,3 ; 1,1,1,1), B_{8}(1,3,3,1 ; 1,1,1,1), \\
B_{8}(2,1,3,2 ; 1,1,1,1), B_{8}(2,3,1,2 ; 1,1,1,1), B_{8}(3,1,3,1 ; 1,1,1,1), \\
B_{8}(3,3,1,1 ; 1,1,1,1), B_{8}(1,1,2,3 ; 1,1,1,2), B_{8}(1,2,2,2 ; 1,1,1,2), \\
B_{8}(1,3,2,1 ; 1,1,1,2), B_{8}(2,1,2,2 ; 1,1,1,2), B_{8}(2,2,2,1 ; 1,1,1,2), \\
B_{8}(3,1,2,1 ; 1,1,1,2), B_{8}(1,1,1,3 ; 1,1,1,3), B_{8}(1,3,1,1 ; 1,1,1,3), \\
B_{8}(2,1,1,2 ; 1,1,1,3), B_{8}(3,1,1,1 ; 1,1,1,3), B_{8}(1,1,3,2 ; 1,1,2,1), \\
B_{8}(1,2,2,2 ; 1,1,2,1), B_{8}(1,3,1,2 ; 1,1,2,1), B_{8}(2,1,3,1 ; 1,1,2,1), \\
B_{8}(2,2,2,1 ; 1,1,2,1), B_{8}(2,3,1,1 ; 1,1,2,1), B_{8}(2,1,1,1 ; 1,1,2,3), \\
B_{8}(1,1,3,1 ; 1,1,3,1), B_{8}(1,3,1,1 ; 1,1,3,1), B_{8}(1,2,1,3 ; 1,2,1,1), \\
B_{8}(1,2,2,2 ; 1,2,1,1), B_{8}(1,2,3,1 ; 1,2,1,1), B_{8}(2,2,1,2 ; 1,2,1,1), \\
B_{8}(2,2,2,1 ; 1,2,1,1), B_{8}(3,2,1,1 ; 1,2,1,1), B_{8}(2,1,1,1 ; 1,2,2,2), \\
B_{8}(2,1,1,2 ; 1,3,1,1), B_{8}(3,1,1,1 ; 1,3,1,1), B_{8}(2,1,1,1 ; 1,3,2,1), \\
B_{8}(2,2,1,2 ; 2,1,1,1), B_{8}(3,1,2,1 ; 2,1,1,1), B_{8}(2,2,1,1 ; 2,1,1,2), \\
B_{8}(3,1,1,1 ; 2,1,1,2), B_{8}(2,1,2,1 ; 2,1,2,1), B_{8}(2,2,1,1 ; 2,1,2,1), \\
B_{8}(3,1,1,1 ; 3,1,1,1) ;\end{array}$ \\
\hline 13 & $\begin{array}{l}B_{8}(1,1,2,5 ; 1,1,1,1), B_{8}(1,1,5,2 ; 1,1,1,1), B_{8}(1,2,1,5 ; 1,1,1,1), \\
B_{8}(1,2,2,4 ; 1,1,1,1), B_{8}(1,2,3,3 ; 1,1,1,1), B_{8}(1,2,4,2 ; 1,1,1,1), \\
B_{8}(1,2,5,1 ; 1,1,1,1), B_{8}(1,3,2,3 ; 1,1,1,1), B_{8}(1,3,3,2 ; 1,1,1,1), \\
B_{8}(1,4,2,2 ; 1,1,1,1), B_{8}(1,5,1,2 ; 1,1,1,1), B_{8}(1,5,2,1 ; 1,1,1,1), \\
B_{8}(2,1,2,4 ; 1,1,1,1), B_{8}(2,1,5,1 ; 1,1,1,1), B_{8}(2,2,1,4 ; 1,1,1,1), \\
B_{8}(2,2,2,3 ; 1,1,1,1), B_{8}(2,2,3,2 ; 1,1,1,1), B_{8}(2,2,4,1 ; 1,1,1,1), \\
B_{8}(2,3,2,2 ; 1,1,1,1), B_{8}(2,3,3,1 ; 1,1,1,1), B_{8}(2,4,2,1 ; 1,1,1,1), \\
B_{8}(2,5,1,1 ; 1,1,1,1), B_{8}(3,1,2,3 ; 1,1,1,1), B_{8}(3,2,1,3 ; 1,1,1,1), \\
B_{8}(3,2,2,2 ; 1,1,1,1), B_{8}(3,2,3,1 ; 1,1,1,1), B_{8}(3,3,2,1 ; 1,1,1,1), \\
B_{8}(4,1,2,2 ; 1,1,1,1), B_{8}(4,2,1,2 ; 1,1,1,1), B_{8}(4,2,2,1 ; 1,1,1,1), \\
B_{8}(5,1,2,1 ; 1,1,1,1), B_{8}(5,2,1,1 ; 1,1,1,1), B_{8}(1,1,1,5 ; 1,1,1,2), \\
B_{8}(1,1,4,2 ; 1,1,1,2), B_{8}(1,2,3,2 ; 1,1,1,2), B_{8}(1,2,4,1 ; 1,1,1,2), \\
B_{8}(1,5,1,1 ; 1,1,1,2), B_{8}(2,1,1,4 ; 1,1,1,2), B_{8}(2,1,4,1 ; 1,1,1,2), \\
B_{8}(2,2,3,1 ; 1,1,1,2), B_{8}(3,1,1,3 ; 1,1,1,2), B_{8}(4,1,1,2 ; 1,1,1,2), \\
B_{8}(5,1,1,1 ; 1,1,1,2), B_{8}(1,1,3,2 ; 1,1,1,3), B_{8}(1,2,3,1 ; 1,1,1,3), \\
B_{8}(2,1,3,1 ; 1,1,1,3), B_{8}(1,1,2,2 ; 1,1,1,4), B_{8}(1,2,2,1 ; 1,1,1,4), \\
B_{8}(2,1,2,1 ; 1,1,1,4), B_{8}(1,2,1,1 ; 1,1,1,5), B_{8}(2,1,1,1 ; 1,1,1,5), \\
B_{8}(1,1,2,4 ; 1,1,2,1), B_{8}(1,1,5,1 ; 1,1,2,1), B_{8}(1,2,1,4 ; 1,1,2,1), \\
B_{8}(1,2,2,3 ; 1,1,2,1), B_{8}(1,5,1,1 ; 1,1,2,1), B_{8}(2,1,2,3 ; 1,1,2,1), \\
B_{8}(2,2,1,3 ; 1,1,2,1), B_{8}(2,2,2,2 ; 1,1,2,1), B_{8}(3,1,2,2 ; 1,1,2,1), \\
B_{8}(3,2,1,2 ; 1,1,2,1), B_{8}(3,2,2,1 ; 1,1,2,1), B_{8}(4,1,2,1 ; 1,1,2,1), \\
B_{8}(4,2,1,1 ; 1,1,2,1), B_{8}(1,1,2,3 ; 1,1,2,2), B_{8}(1,1,3,2 ; 1,1,2,2),\end{array}$ \\
\hline
\end{tabular}


continued from previous page

\begin{tabular}{l|l}
\hline$n$ & $\mathcal{B}^{*}$ \\
\hline \hline & $B_{8}(1,1,4,1 ; 1,1,2,2), B_{8}(2,1,1,3 ; 1,1,2,2), B_{8}(2,1,2,2 ; 1,1,2,2)$, \\
& $B_{8}(2,1,3,1 ; 1,1,2,2), B_{8}(3,1,1,2 ; 1,1,2,2), B_{8}(3,1,2,1 ; 1,1,2,2)$, \\
& $B_{8}(4,1,1,1 ; 1,1,2,2), B_{8}(1,1,3,1 ; 1,1,2,3), B_{8}(2,1,2,1 ; 1,1,2,3)$, \\
& $B_{8}(1,2,1,3 ; 1,1,3,1), B_{8}(2,1,2,2 ; 1,1,3,1), B_{8}(2,2,1,2 ; 1,1,3,1)$, \\
& $B_{8}(3,1,2,1 ; 1,1,3,1), B_{8}(3,2,1,1 ; 1,1,3,1), B_{8}(2,1,1,2 ; 1,1,3,2)$, \\
& $B_{8}(2,1,2,1 ; 1,1,3,2), B_{8}(3,1,1,1 ; 1,1,3,2), B_{8}(1,2,1,2 ; 1,1,4,1)$, \\
& $B_{8}(2,1,2,1 ; 1,1,4,1), B_{8}(2,2,1,1 ; 1,1,4,1), B_{8}(2,1,1,1 ; 1,1,4,2)$, \\
& $B_{8}(1,2,1,1 ; 1,1,5,1), B_{8}(1,3,2,2 ; 1,2,1,1), B_{8}(1,4,1,2 ; 1,2,1,1)$, \\
& $B_{8}(1,4,2,1 ; 1,2,1,1), B_{8}(2,1,1,4 ; 1,2,1,1), B_{8}(2,3,2,1 ; 1,2,1,1)$, \\
& $B_{8}(2,4,1,1 ; 1,2,1,1), B_{8}(3,1,1,3 ; 1,2,1,1), B_{8}(4,1,1,2 ; 1,2,1,1)$, \\
& $B_{8}(5,1,1,1 ; 1,2,1,1), B_{8}(1,2,3,1 ; 1,2,1,2), B_{8}(1,3,2,1 ; 1,2,1,2)$, \\
& $B_{8}(1,4,1,1 ; 1,2,1,2), B_{8}(1,2,2,1 ; 1,2,1,3), B_{8}(1,3,1,2 ; 1,2,2,1)$, \\
& $B_{8}(1,4,1,1 ; 1,2,2,1), B_{8}(2,1,1,3 ; 1,2,2,1), B_{8}(2,2,1,2 ; 1,2,2,1)$, \\
& $B_{8}(2,3,1,1 ; 1,2,2,1), B_{8}(3,1,1,2 ; 1,2,2,1), B_{8}(3,2,1,1 ; 1,2,2,1)$, \\
& $B_{8}(4,1,1,1 ; 1,2,2,1), B_{8}(2,1,1,2 ; 1,2,3,1), B_{8}(2,2,1,1 ; 1,2,3,1)$, \\
& $B_{8}(3,1,1,1 ; 1,2,3,1), B_{8}(2,1,1,1 ; 1,2,3,2), B_{8}(2,1,1,1 ; 1,2,4,1)$, \\
& $B_{8}(1,3,1,2 ; 1,3,1,1), B_{8}(1,3,2,1 ; 1,3,1,1), B_{8}(2,3,1,1 ; 1,3,1,1)$, \\
& $B_{8}(2,2,1,1 ; 1,3,2,1), B_{8}(2,2,1,1 ; 1,4,1,1), B_{8}(2,1,1,1 ; 1,5,1,1)$, \\
& $B_{8}(2,1,1,4 ; 2,1,1,1), B_{8}(2,1,2,3 ; 2,1,1,1), B_{8}(2,1,3,2 ; 2,1,1,1)$, \\
& $B_{8}(2,1,4,1 ; 2,1,1,1), B_{8}(2,2,2,2 ; 2,1,1,1), B_{8}(2,2,3,1 ; 2,1,1,1)$, \\
& $B_{8}(2,3,2,1 ; 2,1,1,1), B_{8}(2,4,1,1 ; 2,1,1,1), B_{8}(3,1,1,3 ; 2,1,1,1)$, \\
& $B_{8}(3,1,2,2 ; 2,1,1,1), B_{8}(3,2,1,2 ; 2,1,1,1), B_{8}(3,2,2,1 ; 2,1,1,1)$, \\
& $B_{8}(3,3,1,1 ; 2,1,1,1), B_{8}(4,1,1,2 ; 2,1,1,1), B_{8}(4,2,1,1 ; 2,1,1,1)$, \\
& $B_{8}(5,1,1,1 ; 2,1,1,1), B_{8}(2,1,1,3 ; 2,1,1,2), B_{8}(2,1,2,2 ; 2,1,1,2)$, \\
& $B_{8}(2,1,3,1 ; 2,1,1,2), B_{8}(2,2,2,1 ; 2,1,1,2), B_{8}(3,1,1,2 ; 2,1,1,2)$, \\
& $B_{8}(2,1,2,1 ; 2,1,1,3), B_{8}(2,1,2,2 ; 2,1,2,1), B_{8}(3,1,1,2 ; 2,1,2,1)$, \\
& $B_{8}(3,2,1,1 ; 2,1,2,1), B_{8}(4,1,1,1 ; 2,1,2,1), B_{8}(3,1,1,1 ; 2,1,2,2)$, \\
& $B_{8}(3,1,1,1 ; 2,1,3,1), B_{8}(2,2,2,1 ; 2,2,1,1), B_{8}(2,3,1,1 ; 2,2,1,1)$, \\
&
\end{tabular}

Table 5: $k=9$.

\begin{tabular}{r|l}
$n$ & $\mathcal{B}^{*}$ \\
\hline \hline \multirow{2}{*}{11} & $B_{9}(2,1,2,1 ; 1,1,1,1 ; 1), B_{9}(2,1,1,1 ; 1,1,1,2 ; 1), B_{9}(1,1,2,1 ; 1,1,2,1 ; 1)$, \\
& $B_{9}(2,1,1,1 ; 2,1,1,1 ; 1) ;$ \\
\hline \multirow{2}{*}{12} & $B_{9}(1,2,1,3 ; 1,1,1,1 ; 1), B_{9}(1,2,3,1 ; 1,1,1,1 ; 1), B_{9}(2,1,2,2 ; 1,1,1,1 ; 1)$, \\
& $B_{9}(2,2,2,1 ; 1,1,1,1 ; 1), B_{9}(3,2,1,1 ; 1,1,1,1 ; 1), B_{9}(1,1,1,3 ; 1,1,1,1 ; 2)$, \\
\hline
\end{tabular}


continued from previous page

\begin{tabular}{|c|c|}
\hline$n$ & $\mathcal{B}^{*}$ \\
\hline 12 & $\begin{array}{l}B_{9}(1,1,3,1 ; 1,1,1,1 ; 2), B_{9}(2,1,1,2 ; 1,1,1,1 ; 2), B_{9}(3,1,1,1 ; 1,1,1,1 ; 2), \\
B_{9}(1,2,1,2 ; 1,1,1,2 ; 1), B_{9}(2,1,1,2 ; 1,1,1,2 ; 1), B_{9}(1,1,2,1 ; 1,1,1,2 ; 2), \\
B_{9}(1,2,1,1 ; 1,1,1,3 ; 1), B_{9}(1,1,2,2 ; 1,1,2,1 ; 1), B_{9}(1,2,1,2 ; 1,1,2,1 ; 1), \\
B_{9}(2,1,1,1 ; 1,1,2,1 ; 2), B_{9}(1,2,1,1 ; 1,1,2,2 ; 1), B_{9}(2,1,1,1 ; 1,1,2,2 ; 1), \\
B_{9}(1,2,1,1 ; 1,1,3,1 ; 1), B_{9}(3,1,1,1 ; 1,2,1,1 ; 1), B_{9}(2,1,1,1 ; 1,2,2,1 ; 1), \\
B_{9}(2,2,1,1 ; 2,1,1,1 ; 1)\end{array}$ \\
\hline 13 & 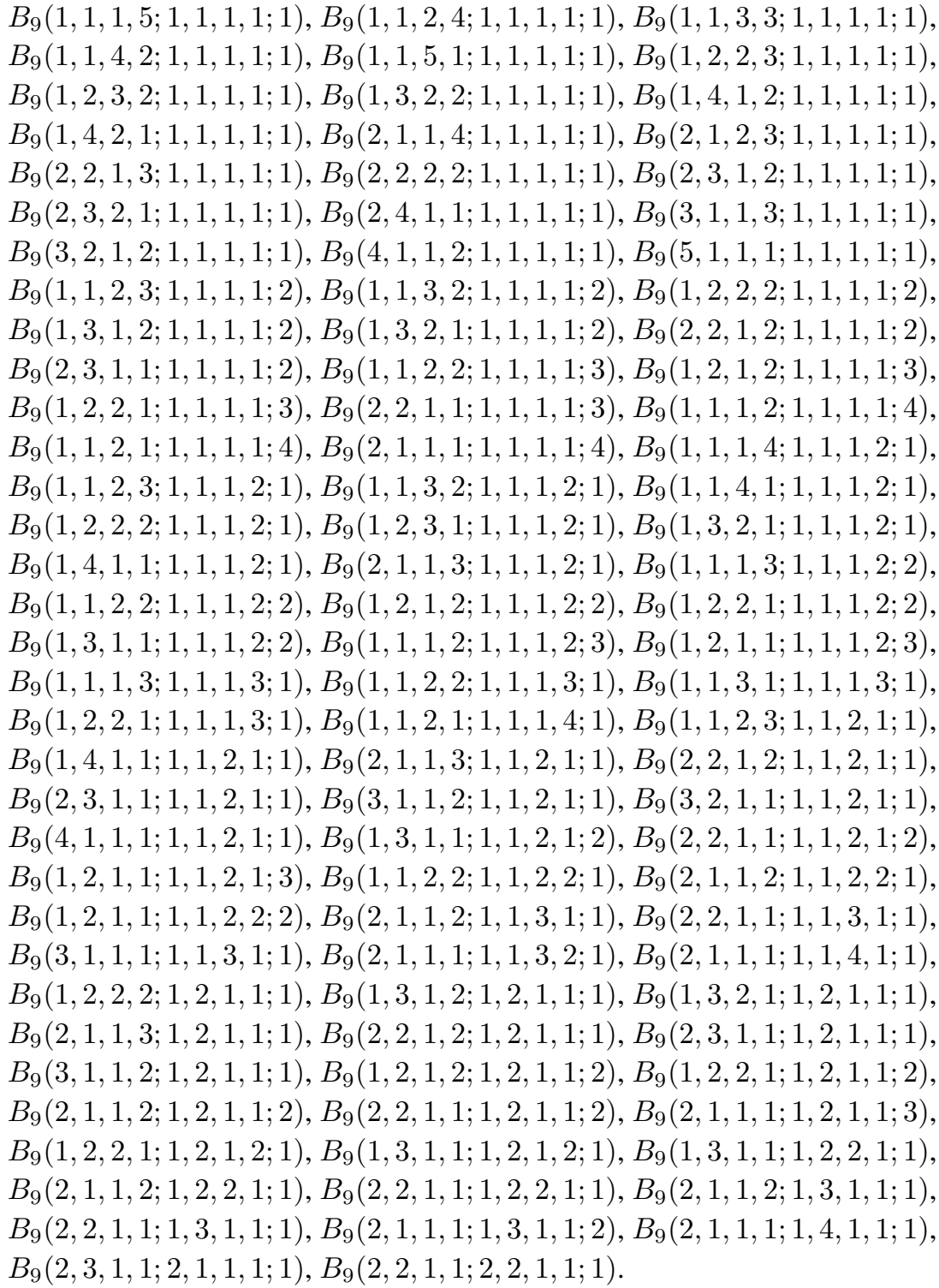 \\
\hline
\end{tabular}


Table 6: $k=10$.

\begin{tabular}{|c|c|}
\hline$n$ & 3* \\
\hline 12 & $\begin{array}{l}B_{10}(1,1,2,1,2 ; 1,1,1,1,1), B_{10}(1,2,1,2,1 ; 1,1,1,1,1), \\
B_{10}(2,1,2,1,1 ; 1,1,1,1,1), B_{10}(1,1,1,1,2 ; 1,1,1,1,2), \\
B_{10}(1,2,1,1,1 ; 1,1,1,1,2), B_{10}(2,1,1,1,1 ; 1,1,1,1,2), \\
B_{10}(1,1,2,1,1 ; 1,1,1,2,1), B_{10}(1,2,1,1,1 ; 1,1,1,2,1), \\
B_{10}(1,1,2,1,1 ; 1,1,2,1,1), B_{10}(2,1,1,1,1 ; 1,2,1,1,1)\end{array}$ \\
\hline 13 & 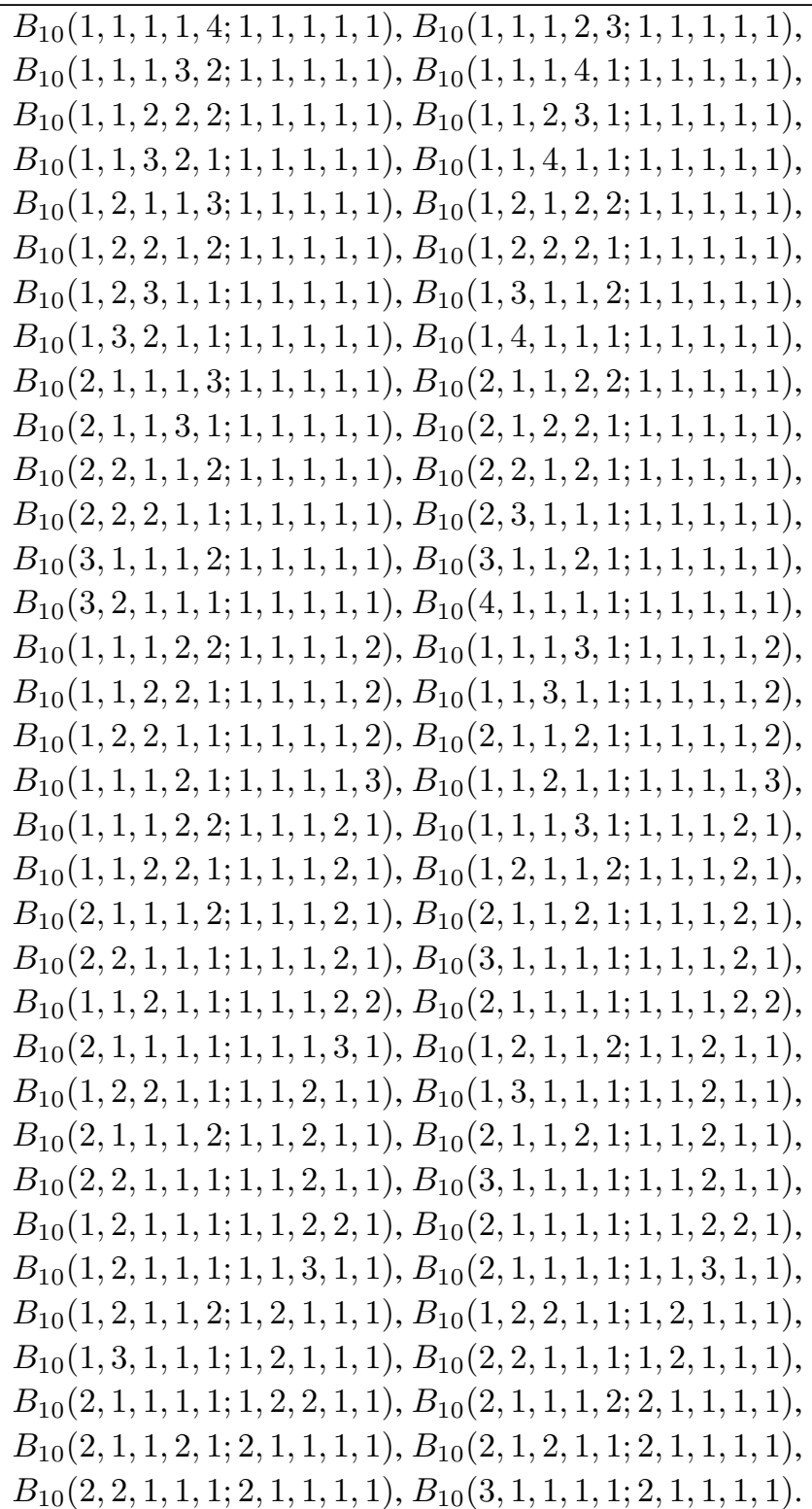 \\
\hline
\end{tabular}




\section{Appendix B Some theorems and lemmas}

Theorem B.1 ([6]). Let $G=B_{4}\left(a_{1}, a_{2} ; a_{3}, a_{4}\right)$, where $a_{1}, a_{2}, a_{3}, a_{4}$ are some positive integers. Then $\lambda_{2}(G)>0$ and $\lambda_{3}(G)<0$ if and only if $G$ is isomorphic to one of the following graphs:
(1) $B_{4}(a, b ; 1, d)$;
(3) $B_{4}(a, 1 ; c, 1)$;
(5) $B_{4}(a, 1 ; x, d)$;
(7) $B_{4}(w, x ; y, d)$;
(2) $B_{4}(a, x ; y, 1)$;
(4) $B_{4}(a, 1 ; w, x)$;
(6) $B_{4}(w, b ; x, 1)$;
(8) $B_{4}(x, b ; y, d)$;
(9) 25 specific graphs: 5 graphs of order 10, 10 graphs of order 11, and 10 graphs of order 12 ,

where $a, b, c, d, x, y, w$ are some positive integers such that $x \leq 2, y \leq 2$ and $w \leq 3$.

Lemma B.2. Let $G \in \mathcal{B}_{4}(n)$, where $n \geq 14$. If $G \notin \mathcal{B}_{4}^{-}(n)$, then $G$ has an induced subgraph $\Gamma \in \mathcal{B}_{4}(14) \backslash \mathcal{B}_{4}^{-}(14)$.

Proof. By the proof of Lemma 5.13, it suffices to prove that $G$ contains an induced subgraph $G^{\prime} \in \mathcal{B}_{4}(n-1) \backslash \mathcal{B}_{4}^{-}(n-1)$ for $n \geq 15$ in the following.

Let $G=B_{4}\left(n_{1}, n_{2} ; n_{3}, n_{4}\right) \in \mathcal{B}_{4}(n)$. Then one of

$$
\begin{aligned}
& H_{1}=B_{4}\left(n_{1}-1, n_{2} ; n_{3}, n_{4}\right), \quad H_{2}=B_{4}\left(n_{1}, n_{2}-1 ; n_{3}, n_{4}\right) \text {, } \\
& H_{3}=B_{4}\left(n_{1}, n_{2} ; n_{3}-1, n_{4}\right) \quad \text { and } \quad H_{4}=B_{4}\left(n_{1}, n_{2} ; n_{3}, n_{4}-1\right)
\end{aligned}
$$

must belong to $\mathcal{B}_{4}(n-1)$. On the contrary, assume that $H_{i} \in \mathcal{B}_{4}^{-}(n-1)(i=1,2,3,4)$. Then $H_{i}$ is a graph belonging to (1) $-(8)$ in Theorem B. 1 since $n \geq 15$.

First we consider $H_{1}$. If $H_{1}$ is a graph belonging to (1) of Theorem B.1, then $H_{1}=$ $B_{4}(a, b ; 1, d)$ where $n_{1}-1=a, n_{2}=b, n_{3}=1$ and $n_{4}=d$, hence $G=B_{4}(a+1, b$; $1, d) \in \mathcal{B}_{4}^{-}(n)$, a contradiction. Similarly, $H_{1}$ cannot belong to (2) - (5) of Theorem B.1. Hence $H_{1}$ is belong to (6) - (8) of Theorem B.1 from which we see that $n_{1}-1$ is either $w$ or $x$. Thus $n_{1} \leq 4$ due to $w \leq 3$ and $x \leq 2$.

By the same method, we can verify that $n_{2} \leq 3$ if $H_{2} \in \mathcal{B}_{4}^{-}(n-1) ; n_{3} \leq 4$ if $H_{3} \in \mathcal{B}_{4}^{-}(n-1)$ and $n_{4} \leq 3$ if $H_{4} \in \mathcal{B}_{4}^{-}(n-1)$. Hence $n=n_{1}+\cdots+n_{4} \leq 14$, a contradiction. We are done.

Theorem B.3 ([6]). Let $G=B_{6}\left(a_{1}, a_{2}, a_{3} ; a_{4}, a_{5}, a_{6}\right)$, where $a_{1}, \ldots, a_{6}$ are some positive integers. Then $\lambda_{2}(G)>0$ and $\lambda_{3}(G)<0$ if and only if $G$ is isomorphic to one of the following graphs:
(1) $B_{6}(a, x, c ; 1,1,1)$;
(6) $B_{6}(x, b, 1 ; y, 1,1)$;
(11) $B_{6}(1, b, 1 ; 1, e, 1)$;
(2) $B_{6}(a, 1, c ; 1, e, 1)$;
(7) $B_{6}(x, y, 1 ; 1, e, 1)$;
(12) $B_{6}(1, b, 1 ; 1, x, y)$;
(3) $B_{6}(a, 1, c ; 1, x, y)$;
(8) $B_{6}(x, y, 1 ; 1,1, f)$;
(13) $B_{6}(1, x, y ; 1,1, f)$;
(4) $B_{6}(a, 1, c ; 1,1, f)$;
(9) $B_{6}(x, 1, c ; y, 1, f)$;
(5) $B_{6}(a, 1,1 ; x, e, 1)$;
(10) $B_{6}(1, b, x ; 1,1,1)$;
(14) 145 specific graphs: 22 graphs of order 10, 54 graphs of order 11, and 69 graphs of order 12 ,

where $a, b, c, d, e, f, x, y$ are some positive integers such that $x \leq 2$ and $y \leq 2$. 
Lemma B.4. Let $G \in \mathcal{B}_{6}(n)$, where $n \geq 14$. If $G \notin \mathcal{B}_{6}^{-}(n)$, then $G$ has an induced subgraph $\Gamma \in \mathcal{B}_{6}(14) \backslash \mathcal{B}_{6}^{-}(14)$.

Proof. By the proof of Lemma 5.13, it suffices to prove that $G$ contains an induced subgraph $G^{\prime} \in \mathcal{B}_{6}(n-1) \backslash \mathcal{B}_{6}^{-}(n-1)$ for $n \geq 15$ in the following.

Let $G=B_{6}\left(n_{1}, n_{2}, n_{3} ; n_{4}, n_{5}, n_{6}\right) \in \mathcal{B}_{6}(n)$. Then one of

$$
\begin{aligned}
& H_{1}=B_{6}\left(n_{1}-1, n_{2}, n_{3} ; n_{4}, n_{5}, n_{6}\right), \quad H_{2}=B_{6}\left(n_{1}, n_{2}-1, n_{3} ; n_{4}, n_{5}, n_{6}\right) \text {, } \\
& H_{3}=B_{6}\left(n_{1}, n_{2}, n_{3}-1 ; n_{4}, n_{5}, n_{6}\right), \quad H_{4}=B_{6}\left(n_{1}, n_{2}, n_{3} ; n_{4}-1, n_{5}, n_{6}\right) \text {, } \\
& H_{5}=B_{6}\left(n_{1}, n_{2}, n_{3} ; n_{4}, n_{5}-1, n_{6}\right) \quad \text { and } \quad H_{6}=B_{6}\left(n_{1}, n_{2}, n_{3} ; n_{4}, n_{5}, n_{6}-1\right)
\end{aligned}
$$

must belong to $\mathcal{B}_{6}(n-1)$. On the contrary, assume that $H_{i} \in \mathcal{B}_{6}^{-}(n-1)(i=1,2, \ldots, 6)$. Then $H_{i}$ is a graph belonging to (1) $-(13)$ in Theorem B.3 since $n \geq 15$.

Let us consider $H_{3}$. If $H_{3}$ is a graph belonging to (1) of Theorem B.3, then $H_{3}=$ $B_{6}(a, x, c ; 1,1,1)$ where $n_{1}=a, n_{2}=x, n_{3}-1=c, n_{4}=n_{5}=n_{6}=1$, hence $G=B_{6}(a, x, c+1 ; 1,1,1) \in \mathcal{B}_{6}^{-}(n)$, a contradiction. Similarly, $H_{3}$ cannot belong to (2) $-(4)$ and (9) of Theorem B.3. If $H_{3}$ is a graph belonging to (10) of Theorem B.3, then $H_{3}=B_{6}(1, b, x ; 1,1,1)$, where $n_{1}=1, n_{2}=b, n_{3}-1=x, n_{4}=n_{5}=n_{6}=1$. Since $x \leq 2$, we have $n_{3} \leq 3$. If $n_{3}<3$ then $x+1 \leq 2$ and $G=B_{6}(1, b, x+1 ; 1,1,1) \in$ $\mathcal{B}_{6}^{-}(n)$, a contradiction. Now assume that $n_{3}=3$. Then $H_{3}=B_{6}(1, b, 2 ; 1,1,1)$, and so $G=B_{6}(1, b, 3 ; 1,1,1)$. By Theorem B.3, $G \notin \mathcal{B}_{6}^{-}(n)$, and also its induced subgraph $B_{6}(1, b-1,3 ; 1,1,1) \notin \mathcal{B}_{6}^{-}(n-1)$, a contradiction. Similarly, $H_{3}$ cannot belong to (13) of Theorem B.3. Hence $H_{3}$ is belong to (5) - (8) and (11)-(12) of Theorem B.3 from which we see that $n_{3}-1 \leq 1$. Thus $n_{3} \leq 2$.

By the same method, we can verify that $n_{1} \leq 3$ if $H_{1} \in \mathcal{B}_{6}^{-}(n-1) ; n_{2} \leq 3$ if $H_{2} \in \mathcal{B}_{6}^{-}(n-1) ; n_{4} \leq 2$ if $H_{4} \in \mathcal{B}_{6}^{-}(n-1) ; n_{5} \leq 2$ if $H_{5} \in \mathcal{B}_{6}^{-}(n-1)$ and $n_{6} \leq 2$ if $H_{6} \in \mathcal{B}_{6}^{-}(n-1)$. Hence $n=n_{1}+\cdots+n_{6} \leq 14$, a contradiction. We are done.

Theorem B.5 ([6]). Let $G=B_{7}\left(a_{1}, a_{2}, a_{3} ; a_{4}, a_{5}, a_{6} ; a_{7}\right)$, where $a_{1}, \ldots, a_{7}$ are some positive integers. Then $\lambda_{2}(G)>0$ and $\lambda_{3}(G)<0$ if and only if $G$ is isomorphic to one of the following graphs:

(1) $B_{7}(a, 1, x ; 1, e, 1 ; 1)$;

(4) $B_{7}(x, y, 1 ; 1, e, 1 ; g)$;

(7) $B_{7}(1, b, 1 ; 1, e, 1 ; g)$;

(2) $B_{7}(a, 1,1 ; 1, e, 1 ; g)$;

(5) $B_{7}(x, 1,1 ; y, 1,1 ; g)$;

(8) $B_{7}(1,1, c ; 1,1, f ; 1)$;

(3) $B_{7}(a, 1,1 ; 1,1, x ; 1)$;

(6) $B_{7}(1, b, x ; 1,1,1 ; g)$;

(9) 143 specific graphs: 18 graphs of order 10, 52 graphs of order 11, and 73 graphs of order 12 ,

where $a, b, c, d, e, f, g, x, y$ are some positive integers such that $x \leq 2$ and $y \leq 2$.

Lemma B.6. Let $G \in \mathcal{B}_{7}(n)$, where $n \geq 14$. If $G \notin \mathcal{B}_{7}^{-}(n)$, then $G$ has an induced subgraph $\Gamma \in \mathcal{B}_{7}(14) \backslash \mathcal{B}_{7}^{-}(14)$.

Proof. By the proof of Lemma 5.13, it suffices to prove that $G$ contains an induced subgraph $G^{\prime} \in \mathcal{B}_{7}(n-1) \backslash \mathcal{B}_{7}^{-}(n-1)$ for $n \geq 15$ in the following.

Let $G=B_{7}\left(n_{1}, n_{2}, n_{3} ; n_{4}, n_{5}, n_{6} ; n_{7}\right) \in \mathcal{B}_{7}(n)$. Then one of

$$
\begin{aligned}
& H_{1}=B_{7}\left(n_{1}-1, n_{2}, n_{3} ; n_{4}, n_{5}, n_{6} ; n_{7}\right), \\
& H_{2}=B_{7}\left(n_{1}, n_{2}-1, n_{3} ; n_{4}, n_{5}, n_{6} ; n_{7}\right), \\
& H_{3}=B_{7}\left(n_{1}, n_{2}, n_{3}-1 ; n_{4}, n_{5}, n_{6} ; n_{7}\right),
\end{aligned}
$$




$$
\begin{aligned}
& H_{4}=B_{7}\left(n_{1}, n_{2}, n_{3} ; n_{4}-1, n_{5}, n_{6} ; n_{7}\right), \\
& H_{5}=B_{7}\left(n_{1}, n_{2}, n_{3} ; n_{4}, n_{5}-1, n_{6} ; n_{7}\right) ; \\
& H_{6}=B_{7}\left(n_{1}, n_{2}, n_{3} ; n_{4}, n_{5}, n_{6}-1 ; n_{7}\right) \text { and } \\
& H_{7}=B_{7}\left(n_{1}, n_{2}, n_{3} ; n_{4}, n_{5}, n_{6} ; n_{7}-1\right)
\end{aligned}
$$

must belong to $\mathcal{B}_{7}(n-1)$. On the contrary, assume that $H_{i} \in \mathcal{B}_{7}^{-}(n-1)(i=1,2, \ldots, 7)$. Then $H_{i}$ is a graph belonging to (1) $-(8)$ in Theorem B.5 since $n \geq 15$.

Let us consider $H_{1}$. If $H_{1}$ is a graph belonging to (1) of Theorem B.5, then $H_{1}=$ $B_{7}(a, 1, x ; 1, e, 1 ; 1)$ where $n_{1}-1=a, n_{2}=1, n_{3}=x, n_{4}=1, n_{5}=e, n_{6}=n_{7}=1$, hence $G=B_{7}(a+1,1, x ; 1, e, 1 ; 1) \in \mathcal{B}_{7}^{-}(n)$, a contradiction. Similarly, $H_{1}$ cannot belong to (2) - (3) of Theorem B.5. If $H_{1}$ is a graph belonging to (4) of Theorem B.5, then $H_{1}=B_{7}(x, y, 1 ; 1, e, 1 ; g)$, where $n_{1}-1=x, n_{2}=y, n_{3}=n_{4}=1, n_{5}=e$, $n_{6}=1$ and $n_{7}=g$. Since $x \leq 2$, we have $n_{1} \leq 3$. If $n_{1}<3$ then $x+1 \leq 2$ and $G=B_{7}(x+1, y, 1 ; 1, e, 1 ; g) \in \mathcal{B}_{7}^{-}(n)$, a contradiction. Now assume that $n_{1}=3$. Then $H_{1}=B_{7}(2, y, 1 ; 1, e, 1 ; g)$, and so $G=B_{7}(3, y, 1 ; 1, e, 1 ; g)$. Since $y \in\{1,2\}$, we have $G \in\left\{B_{7}(3,1,1 ; 1, e, 1 ; g), B_{7}(3,2,1 ; 1, e, 1 ; g)\right\}$. However $B_{7}(3,1,1 ; 1, e, 1 ; g)$ belongs to (2) of Theorem B.5 which contradicts our assumption. Thus $G=B_{7}(3,2,1 ; 1, e, 1 ; g)$. By Theorem B.5, $G \notin \mathcal{B}_{7}^{-}(n)$, and also its induced subgraph $B_{7}(3,2,1 ; 1, e-1,1 ; g)$ or $B_{7}(3,2,1 ; 1, e, 1 ; g-1)$ is not in $\mathcal{B}_{7}^{-}(n-1)$, a contradiction. Similarly, $H_{1}$ cannot belong to (5) of Theorem B.5. Hence $H_{1}$ belongs to (6) - (8) of Theorem B.5 from which we see that $n_{1}-1 \leq 1$. Thus $n_{1} \leq 2$.

By the same method, we can verify that $n_{2} \leq 2$ if $H_{2} \in \mathcal{B}_{7}^{-}(n-1) ; n_{3} \leq 2$ if $H_{3} \in \mathcal{B}_{7}^{-}(n-1) ; n_{4} \leq 2$ if $H_{4} \in \mathcal{B}_{7}^{-}(n-1) ; n_{5} \leq 2$ if $H_{5} \in \mathcal{B}_{7}^{-}(n-1), n_{6} \leq 2$ if $H_{6} \in \mathcal{B}_{7}^{-}(n-1)$ and $n_{7} \leq 2$ if $H_{7} \in \mathcal{B}_{7}^{-}(n-1)$. Hence $n=n_{1}+\cdots+n_{7} \leq 14$, a contradiction. We are done.

Theorem B.7 ([6]). Let $G=B_{8}\left(a_{1}, a_{2}, a_{3}, a_{4} ; a_{5}, a_{6}, a_{7}, a_{8}\right)$, where $a_{1}, \ldots, a_{8}$ are some positive integers. Then $\lambda_{2}(G)>0$ and $\lambda_{3}(G)<0$ if and only if $G$ is isomorphic to one of the following graphs:

(1) $B_{8}(a, 1,1, d ; 1,1, g, 1)$;

(2) $B_{8}(1, b, 1,1 ; 1, f, 1,1)$;

(3) 134 specific graphs: 12 graphs of order 10, 42 graphs of order 11, and 80 graphs of order 12 ,

where $a, b, d, f, g$ are some positive integers.

Lemma B.8. Let $G \in \mathcal{B}_{8}(n)$, where $n \geq 14$. If $G \notin \mathcal{B}_{8}^{-}(n)$, then $G$ has an induced subgraph $\Gamma \in \mathcal{B}_{8}(14) \backslash \mathcal{B}_{8}^{-}(14)$.

Proof. By the proof of Lemma 5.13, it suffices to prove that $G$ contains an induced subgraph $G^{\prime} \in \mathcal{B}_{8}(n-1) \backslash \mathcal{B}_{8}^{-}(n-1)$ for $n \geq 15$ in the following.

Let $G=B_{8}\left(n_{1}, n_{2}, n_{3}, n_{4} ; n_{5}, n_{6}, n_{7}, n_{8}\right) \in \mathcal{B}_{8}(n)$ and

$$
\begin{aligned}
& H_{1}=B_{8}\left(n_{1}-1, n_{2}, n_{3}, n_{4} ; n_{5}, n_{6}, n_{7}, n_{8}\right), \\
& H_{2}=B_{8}\left(n_{1}, n_{2}-1, n_{3}, n_{4} ; n_{5}, n_{6}, n_{7}, n_{8}\right), \\
& H_{3}=B_{8}\left(n_{1}, n_{2}, n_{3}-1, n_{4} ; n_{5}, n_{6}, n_{7}, n_{8}\right), \\
& H_{4}=B_{8}\left(n_{1}, n_{2}, n_{3}, n_{4}-1 ; n_{5}, n_{6}, n_{7}, n_{8}\right), \\
& H_{5}=B_{8}\left(n_{1}, n_{2}, n_{3}, n_{4} ; n_{5}-1, n_{6}, n_{7}, n_{8}\right),
\end{aligned}
$$




$$
\begin{aligned}
& H_{6}=B_{8}\left(n_{1}, n_{2}, n_{3}, n_{4} ; n_{5}, n_{6}-1, n_{7}, n_{8}\right), \\
& H_{7}=B_{8}\left(n_{1}, n_{2}, n_{3}, n_{4} ; n_{5}, n_{6}, n_{7}-1, n_{8}\right) \quad \text { and } \\
& H_{8}=B_{8}\left(n_{1}, n_{2}, n_{3}, n_{4} ; n_{5}, n_{6}, n_{7}, n_{8}-1\right) .
\end{aligned}
$$

If $n_{3} \geq 3$, then $H_{3} \in \mathcal{B}_{8}(n-1) \backslash \mathcal{B}_{8}^{-}(n-1)$ by Theorem B.7 as desired. If $n_{3}=2$, then at least one of $n_{1}, n_{2}, n_{4}, n_{5}, n_{6}, n_{7}, n_{8}$ is greater than 1 since $n \geq 15$, say $n_{2}$. Thus $H_{2} \in \mathcal{B}_{8}(n-1) \backslash \mathcal{B}_{8}^{-}(n-1)$ by Theorem B.7 as desired. Hence let $n_{3}=1$. Similarly, let $n_{5}=n_{8}=1$. Thus one of $H_{1}, H_{2}, H_{4}, H_{6}, H_{7}$ must belong to $\mathcal{B}_{8}(n-1)$. On the contrary, assume that $H_{i} \in \mathcal{B}_{8}^{-}(n-1)(i=1,2,4,6,7)$. Then $H_{i}$ is a graph belonging to (1) - (2) in Theorem B.7 since $n \geq 15$.

Let us consider $H_{1}$. If $H_{1}$ is a graph belonging to (1) of Theorem B.7, then $H_{1}=$ $B_{8}(a, 1,1, d ; 1,1, g, 1)$; where $n_{1}-1=a, n_{2}=n_{3}=1, n_{4}=d, n_{5}=n_{6}=1, n_{7}=g$ and $n_{8}=1$, hence $G=B_{8}(a+1,1,1, d ; 1,1, g, 1) \in \mathcal{B}_{8}^{-}(n)$, a contradiction. Hence $H_{1}$ belongs to (2) of Theorem B.7 from which we see that $n_{1}=2$ due to $n_{1}-1=1$.

By the same method, we can verify that $n_{i}=2$ if $H_{i} \in \mathcal{B}_{8}^{-}(n-1)$ for $i=2,4,6,7$. Hence $n=n_{1}+\cdots+n_{8} \leq 13$, a contradiction. We are done.

Theorem B.9 ([6]). Let $G=B_{9}\left(a_{1}, a_{2}, a_{3}, a_{4} ; a_{5}, a_{6}, a_{7}, a_{8} ; a_{9}\right)$, where $a_{1}, \ldots, a_{9}$ are some positive integers. Then $\lambda_{2}(G)>0$ and $\lambda_{3}(G)<0$ if and only if $G$ is isomorphic to one of the following graphs:

(1) $B_{9}(1, b, 1,1 ; 1, f, 1,1 ; k)$;

(2) 59 specific graphs: 3 graphs of order 10, 17 graphs of order 11, and 39 graphs of order 12 ,

where $b, f, k$ are some positive integers.

Lemma B.10. Let $G \in \mathcal{B}_{9}(n)$, where $n \geq 14$. If $G \notin \mathcal{B}_{9}^{-}(n)$, then $G$ has an induced subgraph $\Gamma \in \mathcal{B}_{9}(14) \backslash \mathcal{B}_{9}^{-}(14)$.

Proof. By the proof of Lemma 5.13, it suffices to prove that $G$ contains an induced subgraph $G^{\prime} \in \mathcal{B}_{9}(n-1) \backslash \mathcal{B}_{9}^{-}(n-1)$ for $n \geq 15$ in the following.

Let $G=B_{9}\left(n_{1}, n_{2}, n_{3}, n_{4} ; n_{5}, n_{6}, n_{7}, n_{8} ; n_{9}\right) \in \mathcal{B}_{9}(n)$. On the contrary, suppose that every induced subgraphs $G^{\prime} \in \mathcal{B}_{9}(n-1)$ of $G$ belongs to $\mathcal{B}_{9}^{-}(n-1)$. If $n_{1} \geq 3$, then $H_{1}=B_{9}\left(n_{1}-1, n_{2}, n_{3}, n_{4} ; n_{5}, n_{6}, n_{7}, n_{8} ; n_{9}\right) \notin \mathcal{B}_{9}^{-}(n-1)$ by Theorem B.9, a contradiction. If $n_{1}=2$, then at least one of $n_{2}, n_{3}, n_{4}, n_{5}, n_{6}, n_{7}, n_{8}, n_{9}$ is greater than 1 since $n \geq 15$, say $n_{2}$. Thus $H_{2}=B_{9}\left(n_{1}, n_{2}-1, n_{3}, n_{4} ; n_{5}, n_{6}, n_{7}, n_{8} ; n_{9}\right) \notin \mathcal{B}_{9}^{-}(n-1)$ by Theorem B.9, a contradiction. Hence $n_{1}=1$. Similarly, $n_{3}=n_{4}=n_{5}=n_{7}=n_{8}=$ 1. But now $G=B_{9}\left(1, n_{2}, 1,1 ; 1, n_{6}, 1,1 ; n_{9}\right) \in \mathcal{B}_{9}^{-}(n)$ by Theorem B.9, a contradiction. We are done. 